\title{
Dicer1 is reduced in APPswe/PSEN1dE9 mice and is regulated by Nrf2
}

Yan Wang ${ }^{a, b}$, Meiling Lian ${ }^{a, b}$, Jing Zhou ${ }^{a, b}$, Shengzhou $W^{*}$ a,b

Yan Wang and Meiling Lian equally contributed to the work

${ }^{a}$ School of Optometry and Ophthalmology and the Eye Hospital, Wenzhou Medical University; ${ }^{b}$ State Key Laboratory of Optometry, Ophthalmology, and Visual Science, 270 Xueyuan Road, Wenzhou, Zhejiang, 325003, P.R.China

Correspondence should be directed to:

Prof. Shengzhou Wu, Ph.D, M.D

School of Optometry and Ophthalmology

Wenzhou Medical University

Email: wszlab@wmu.edu.cn or wszlab@163.com

Phone: (86)-577-88067974

Fax: (86)-577-88067934

Manuscript information:The m.s includes 7 figures and 3 supplemental figures, 44 pages, 199 words for Abstract, 350 words for Introduction, and 639 words for Discussion.

Running title: Dicer1 is reduced in AD mice and regulated by Nrf2

Conflict of Interests: All the authors have found no conflict of interests to disclose.

Acknowledgements: This study is supported by Natural Science Foundation of

Zhejiang Province (LY18H120003) and by funding (QTJ10001) from Wenzhou Medical University. 


\section{Abstract}

The pathogenesis of Alzheimer's disease $(A D)$ involves the central roles of oxidative stress. Oxidative stress due to Dicer1 depletion may underline the neurodegeneration in the central nervous system and degeneration of retinal pigment epithelial cells in geographic atrophy form of age-related macular degeneration. We hypothesized that Dicer1 may play roles in AD pathogenesis. Indeed, Dicer1 was reduced in the hippocampus and cortex of APPswe/PSEN1dE9 mice, an AD model. Dicer1 knockdown induced oxidative stress, mitochondrial dysfunction, apoptosis in cultured neurons, and increased secretions of interleukin-1 $1 /-18$, indicators of inflammasome activation. Accordingly, Dicer1 was decreased by amyloid peptide and the effect was connected with down-regulation of nuclear factor erythroid 2related factor 2 (Nrf2). Anti-oxidant response elements (AREs) were identified in the promoter of Dicer1 and Keap1-Nrf2-AREs signaling was demonstrated to regulate Dicer1 expression. Furthermore, overexpression of Dicer1 carried by adenovirus in the cultured neurons rescued neurite deficit induced by amyloid peptide. In consistent with the in vitro results, injection of Dicer1-overexperssion adenovirus in the hippocampus of the AD mice significantly improved spatial learning. Altogether, we unveiled an unexploited roles of Dicer1 in AD and a novel way of Dicer1 regulation. These findings suggest that Dicer1 may be a target in AD therapy. 


\section{Significance Statement}

Dicer1 is a microRNA-processing enzyme, which is central to microRNA maturation. For the first time, we herein reported that Dicer1 was reduced in the hippocampus or the cortex of $A D$ mice before overt amyloid plque deposition and overexpression of Dicer1 in the hippocampus significantly improved spatial learning in AD mice. We also demonstrated that Dicer1 was regulated by Keap1-Nrf2-ARE signaling which is unreported before. These findings advance understandings of $A D$ pathogenesis and suggest that Dicer1 may be a molecular target in AD therapy. 


\section{Introduction}

Alzheimer's disease $(A D)$ is an inexorable neurodegenerative disorder, characterized with extracellular amyloid plaque deposition, intracellular neurofibrillary tangle formation, and extensive neuronal loss (Hardy and Selkoe, 2002; Mattson, 2004).

The pathogenesis of $A D$ involves the key component of oxidative stress which is dependent on the balance between reactive species and anti-oxidation systems (Halliwell, 2006). Accumulation of reactive oxygen/nitrogen species generates oxidative stress and produces ensued cytotoxicity(Markesbery, 1997) as well as does ferroptosis due to iron accumulation in AD brain(Belaidi and Bush, 2016; Stockwell et al., 2017). Amyloid peptide inactivates membrane channel and transporter including sodium/calcium exchanger, calcium ATPase, glutamate and glucose transporter, these hazardous effects involving the roles of ROS and lipid peroxidation(Mark et al., 1995; Keller et al., 1997; Mark et al., 1997). Anti-oxidation systems are used to cope with oxidative injury, in which the regulation of the key enzymes synthesizing glutathione and of the protein supporting the generation and recovery of peroxiredoxins revolves around a transcriptional factor, nuclear factor erythroid 2-related factor 2 (Nrf2)(McWalter et al., 2004; Chen et al., 2005). Nrf2 is a basic leucine zipper protein that regulates expression of a multitude of antioxidant proteins in response to oxidative injuries and is retained in cytoplasm by Keap1 under quiescent situation; upon oxidative stimulation, Nrf2 is released from the binding and translocated into nucleus, triggering the transcription of antioxidant genes(Kobayashi et al., 2004).

Dicer1 is a pre-microRNA processing enzyme which is central to microRNA maturation (Bernstein et al., 2001). Dicer1 is reduced in retinal pigment epithelial cell 
(RPE) which induces cytotoxicity via Alu RNA accumulation, resulting in oxidative injury via activation of NLRP3 inflammasome (Kaneko et al., 2011; Tarallo et al., 2012); upregulation of NLRP3 inflammasome is also observed in Alzheimer's brain (Heneka et al., 2013). In addition of the cytotoxicity induced by Dicer1 depletion in the RPEs, Dicer1 loss induces neurotoxicity in different brain regions (Davis et al., 2008; Chmielarz et al., 2017). In light of these evidence, we exploited the roles of Dicer1 in AD and the regulation of Dicer1 expression. We further explored potential therapy by overexpressing Dicer1 in the hippocampus of APPswe/PSEN1dE9 (APP/PS1) mice. 


\section{Materials and Methods}

\section{Materials}

The following primary antibodies were used in this study: Dicer1(Sigma, St Louis, MO, USA, cat\# WH0023405M1, research resource identifier, RRID: AB_1841286), caspase 3 (Santa Cruz Biotechnology, Santa Cruz, CA, USA, cat\# sc-271759, RRID: AB_10709891), Keap 1(Proteintech, Suzhou, China, cat\# 60027-1-Ig, RRID:

AB_2132623), and rabbit polyclonal anti-Nrf2 (Proteintech, cat\# 16396-1-AP, RRID:AB_2782956). Activated caspase 3 (Beyotime Biotechnology, Haimen, China, cat\# AC033), ßIII-tubulin (Beyotime Biotechnology, cat\# AT809), Histone 3 (Beyotime Biotechnology, cat\# AF0009), Vimentin (Beyotime Biotechnology, cat\# AF1975), GAPDH (Bioworld, Nanjing, China, cat\# MB001), NeuN (Beyotime Biotechnology, cat\# AF1072). The following secondary antibodies were also used in this study: goat antimouse horseradish peroxidase conjugated IgG (Boster Biological Technology, Co. Ltd, Wuhan, China), goat anti-Rabbit horseradish peroxidase conjugated IgG (Boster Biological Technology). The following reagents or cell lines were used in this study: a human Aß42 peptide (GenScript, Nanjing, China, cat\# RP10017), the Dicer1 siRNA duplex and the negative control (NC) siRNA duplex (Genepharma, Suzhou, China), MTS reagent (CellTiter 96 AQueous One Solution, Promega, Beijing, China, cat\# 3580), pfu High fidelity enzyme (Qiagen, Beijing, China, cat\# KP202), pJet1.2 vector (Thermo Fisher Scientific, Carlsbad, CA,USA, cat\# K1231), pGL6-basic (Beyotime Biotechnology, Haimen, China, cat\# D2105), pRL-TK Vector (Beyotime Biotechnology, Inc, cat\# D2762), a human pCMV-Nrf2 (Sino Biological, Beijing, China, cat\# HG17384-U), a mouse pCMV-Nrf2 (Sino Biological, Beijing, China, cat\# MG56971-UT), or an empty 
pCMV3 vector (Sino Biological, Beijing, China, cat\# D2602), mitochondrial extraction kit (Beyotime Biotechnology, cat\# C3601), 2',7'-Dichlorodihydrofluorescein diacetate (Thermo fisher, Shanghai, China, cat\# 2938), JC-1 probe solution (Sigma, St Louis, MO, USA, cat\# CS0760), Neuro-2a (N2A)( ATCC, Manassas, VA cat\# CCL-131, RRID:CVCL_0470), SK-N-BE(2) (ATCC Cat\# CRL-2271, RRID:CVCL_0528).

\section{Animal}

All mice were fed water and food ad libitum in a temperature- and humiditycontrolled animal facility with an automatic illumination on a 12-h on/off cycle in Wenzhou Medical University. All experiments and data reports followed the SfN Policy on Ethics and the study was approved by the Animal Care and Use Committee of Wenzhou Medical University (Approval number\# wydw2019-0141). APPswe/PSEN1dE9 mice (Jackson Laboratory, stock number 004462) express a K595N/M586L Swedish mutations and a mutant human presenilin1 with deletion of exon9 under the control of mouse prion promoter. The mice were multiplied and genotyped according to the guidance by Jackson Laboratory. Both genders of 4- and 11-month-old transgenic mice were used and WT littermates were used as controls. Behavioral experiments were performed during the daytime and at the same time of each day.

\section{Intrahippocampal injection of adenovirus expressing Dicer1}


The adenovirus (Ad) incorporating sequences of either Ad-pCMV-EGFP or AdpCMV-Dicer1:T2A:EGFP in which the transcription of Dicer1 and EGFP is driven by independent promoter were packaged and generated by Cyagen Biosciences Inc. (Guangzhou, China). Before stereotactic injection, animals were anaesthetized by intraperitoneal injection of ketamine/xylazine $(0.1 / 0.05 \mathrm{mg} / \mathrm{g}$ body weight) and mounted on a stereotactic frame (KOPF, KD Scientific). The virus were injected into bilateral hippocampus with $2 \mu \mathrm{l}$ of viral titre $\left(1.2 \times 10^{9} \mathrm{vg} / \mathrm{mL}\right)$ in each hemisphere using a $10-\mu \mathrm{l}$ Hamilton syringe (Hamilton Medical, Reno, NV, USA) connected to a 30-gauge micropipette at an injection rate of $0.2 \mu \mathrm{l} / \mathrm{min}$. The injection coordinates were anteriorposterior, $-2 \mathrm{~mm}$, mediolateral, $\pm 2 \mathrm{~mm}$, dorsoventral, $-2 \mathrm{~mm}$ from bregma. The person in charge of injection wore protective equipment and the injections were conducted in a biological safety cabinet in a biological safety level II lab setting. Autoclave of disposals was used to prevent contamination. The injections were conducted in 3.5-/4-month-old mice and behavior test was performed during 17-23 days after injection in which learning curve was acquired during 17-22 days and probe trial conducted at the 23rd day after injection. Halves of mice were used for immunohistochemistry and halves of them used for western blot.

\section{Morris Water Maze}

Water maze tests were performed using three groups of mice, WT mice (with intrahippocampal injection of Ad-EGFP) and APP/PS1 mice (intrahippocampal injection of Ad-EGFP or Ad-Dicer1-T2A:EGFP). The water maze (1.2-m diameter) was filled 
with water $\left(24^{\circ} \mathrm{C}\right)$ and made opaque by the addition of nontoxic white paint. The water maze was surrounded by a black curtain (placed $80 \mathrm{~cm}$ away) that held three salient visual cues. Initially, mice were randomly trained in four quadrants of the water maze, and were allowed a maximum of $60 \mathrm{~s}$ to find a hidden platform (10-cm diameter, $1 \mathrm{~cm}$ under the water surface). Mice were trained for 6 days (4 trials per day, 10-min interval between trial). On day 7 , mice were given a 60 -s probe test (scanning the platform) to test their spatial memory. $\quad$ SLY-WMS Morris Water Maze System (Beijing Sunny Instruments Co. Ltd) consisting of a frame grabber, a video camera, a water pool, and the analysis software was used to monitor the animal's swimming pattern, distance, speed, and the amount of time spent in each of the four quadrants.

\section{Cell culture}

Newly born mice were used for culturing dissociated cortical and hippocampal neurons (CNs and HNs, respectively). Cortex or hippocampal tissue was mechanically dissociated after digestion with 0.25\% trypsin (Beyotime, Haimen, China) and 0.1\% DNasel (Takara, Dalian, China) at $37^{\circ} \mathrm{C}$ for $15 \mathrm{~min}$. DMEM/F12 containing $10 \%$ fetal bovine serum (Gibco, Billings, MA,USA) was then added, and the cells were centrifuged at $120 \mathrm{~g}$ for $3 \mathrm{~min}$ and the supernatants were then plated on poly-D-lysine-coated $8 \mathrm{~cm}$ culture dishes in neurobasal medium supplemented with 2\% B27 (Invitrogen, Carlsbad, CA,USA) and $10 \mu \mathrm{M}$ cytosine arabinoside (Sigma-Aldrich, Saint Louis, MO, USA). After 24 hours, the culture medium was replaced with fresh neurobasal medium supplemented with 2\% B27 and the neuronal cultures were used for experiments after 
three days in vitro culture (DIV 3). Before transfection, the medium was replaced with DMEM/F12 containing $0.1 \%$ bovine serum albumin. After $24 \mathrm{~h}$ in culture, neurons were used for transfection, Aß42 oligomer treatment or adenovirus infection. N2A, SK-N-BE(2) or HEK 293T cells was cultured in Dulbecco's modified Eagle's Medium(DMEM) containing 10\% Fetal bovine serum(FBS).

\section{Plasmid construction and Luciferase reporter assay}

With genomic DNA extracted from HEK 293T cells as a template, the region upstream of Dicer1 transcription start site (tss) (Entrez acc.no.:NM_177438.2) were amplified, producing an amplimer containing sequence from -1230 (relative to tss) to +3 (promoter 3), using forward primer

CGACGCGTTTGCAGTGAGCTGAGATTGCGCCAC-TACATT and reverse primer CCCAAGCTTCCCAAGCTTCCCAAGCTTCTTTGTGTCC. Two more regions were amplified spanning -1131 or -756 to +3 by alternating forward primer CGACGCGTAGCCTGGGTGACAGAGCGAAACTCT (promoter 2) or CGACGCGTACCTGAGCTGGCTGGGACCCAGCATTTA (promoter 1), respectively. The bold letters indicated the predicted AREs sites with consensus sequence TGA(C/G)NNNGC. A sequence not containing AREs site was also amplified by changing a forward primer CGACGCGTGGGCAGTCAGAGAGAGAGGAAAGGAAGG spanning -581 to +3 (promoter 0 ). The region spanning individual ARE sequence was also amplified by using forward primer CGACGCGTAGGAGATCAAGACCATCCTGGG 
and reverse primer CCCAAGCTTTAGTGGCGCAATCTCAGCT for ARE3 (124 bp).

ARE2 (192 bp) was amplified using forward primer CGACGCGTCAGCCTGGGTGA

CAGAGCGAAAC and reverse primer CCCAAGCTTCTGGTCTGCAAGGCA.

ARE1(185 bp) was amplified using forward primer CGGCACGCGTGAGCTGCC

TTGTGACTTTGCCTTC and reverse primer CCCAAGCTTCGTCTTTCAACACTTGA

-TCA . PCR products were amplified by pfu High fidelity enzyme and cloned into a

pJet1.2 vector and then subcloned into pGL6-basic containing firefly luciferase coding

sequence. The constructs spanning tss were referred to as Dicer 1 promoter 3-Luc,

promoter 2-Luc, promoter 1-Luc, and promoter 0-Luc and the constructs containing individual AREs were referred to as ARE3-Luc, ARE2-Luc, and ARE1-Luc. ARPE1 in promoter 1-Luc was mutated with forward primer incorporating mutated base pair in bold letters GTACCTGGCTGGGACCCAGCATTTA and reverse primer CCCAAGCTTCCCAAGCTTCTTTGTGTCC; promoter 1-Luc was used as the template. Using the resultant plasmid as the template, the forward primer incorporating Mlu I restrict site and the reverse primer containing HindIII restriction site were used to generate product, which was subcloned into PGL6-basic, namely, mutant promoter-1. The forward primer was CGACGCGTACCGTACCTGGCTGGGACCCAGCATTTA and the reverse primer was CCCAAGCTTCCCAAGCTTCCCAAGCTTCTTTGTGTCC with restriction sites underlined.

The HEK293T cells were seeded at a density of $3 \times 10^{4}$ cells/per 48 wells and transfected according to the manufacturer's protocol (Lipofectamine 2000, Invitrogen). The Renilla luciferase-containing reporter (pRL-TK Vector) was mixed with individual 
construct containing firefly luciferase, and a human pCMV-Nrf2 or an empty pCMV3 vector at a ratio of 1:20:20, respectively. The relative luminescence unit (RLU) of luciferase/renilla assay was measured per the manufacturer's protocol (Beyotime Biotechnology, cat\#RG027) by a plate reader (SpectraMax M5, Molecular devices, San Jose, CA, USA ).

\section{A 342 oligomer preparation}

The oligomer form of $A \beta 42$ was prepared as described (Rushworth et al., 2013). Briefly, the peptide was dissolved in 1,1,1,3,3,3-Hexafluoro-2-propanol (Sigma-Aldrich) to remove any aggregates, stored in $4^{\circ} \mathrm{C}$ for $30 \mathrm{~min}$, dried under room temperature,and dissolved in dimethyl sulfoxide (DMSO, Sigma-Aldrich) to $1 \mathrm{mM}$. The dissolved peptide was diluted in phenol-free F12 medium to $500 \mathrm{nM}$ and incubated at $4^{\circ} \mathrm{C}$ for $24 \mathrm{~h}$. The solution was subjected to centrifugation at $14,000 \times \mathrm{g}$ for $30 \mathrm{~min}$ at $4^{\circ} \mathrm{C}$. The supernatant was collected as the oligomer preparation which was used at a final concentration of $100 \mathrm{nM}$.

\section{Cell viability assay}

Neurons were cultured in 96 -well plates at a density of $5 \times 10^{3}$ per well in DMEM/F12 medium supplemented with B-27. After transfected with Dicer1 siRNA duplex (sense, 5'-GCACAUCAAGGUGCUACUATT-3', antisense, 5'UAGUAGCACCUUGAUGUGCTT- 
3' ) or negative control siRNA duplex (sense, 5'-UUCUCCGAACGUGUCACGUTT-3', antisense, 5'-ACGUGACACGUUCGGAGAATT-3' ) by lipofectamine 2000 for $12 \mathrm{~h}$, the medium was replaced with fresh DMEM/F-12 medium and continued to culture for $36 \mathrm{~h}$. At the end of treatment, each well was added with $10 \mu \mathrm{L}$ MTS reagent $(500 \mu \mathrm{g} / \mathrm{mL})$ for 4 h. The absorption values were read in a plate reader at 490nm (SpectraMax M5, Molecular devices).

\section{Measurement of mitochondrial membrane potential and reactive oxygen species (ROS) activity}

Primary cultured cortical neurons at $9 \times 10^{5}$ cells/well and hippocampal neurons at $5 \times 10^{5}$ cells/ well were plated into 6 -well plates and cultured until DIV3. The neurons were transfected with Dicer1 duplex siRNAs (50 pM) per well by lipofectamine 2000 for $12 \mathrm{~h}$ in DMEM/F12 plus 2\% B27. After transfection, the cultured medium was replaced with fresh neurobasal medium supplemented with 2\% B27 and continued to culture for $36 \mathrm{~h}$. For mitochondrial membrane potential $\left(\Delta \Psi_{\mathrm{m}}\right)$ assay, the neurons at the end of treatment were stained with $\mathrm{JC}$-1probe solution at $37^{\circ} \mathrm{C}$ for 20 min and observed under fluorescence microscope (DMi8, Leica Biosystems, Wetzlar, Germany). The mitochondria were also extracted by a mitochondrial extraction kit. Neural mitochondrion $(30 \mu \mathrm{g})$ from each group was incubated with $\mathrm{JC}-1$ probe solution at $37^{\circ} \mathrm{C}$ for $20 \mathrm{~min}$. The reaction product was added into a 96-well plate for fluorescence measurement, which was read with excitation at $490 \mathrm{~nm}$ and emission at $520 \mathrm{~nm}$ for 
monomer (green) and with excitation at $530 \mathrm{~nm}$ and emission at $590 \mathrm{~nm}$ for aggregates (red). The $\Delta \Psi_{\mathrm{m}}$ values were represented by the ratios between aggregate and monomer fluorescence in relative fluorescence units ( RFU). For reactive oxygen species (ROS) assay, CNs and HNs were incubated with $10 \mu \mathrm{M}$ 2', 7'-

Dichlorodihydrofluorescein diacetate in neurobasal medium supplemented with 2\% B27. After incubation at $37^{\circ} \mathrm{C}$ for $30 \mathrm{~min}$, the neurons were added into 96 -well plate at the density of $4 \times 10^{4}$ per well and subjected to measurement with plate fluorescence reader at 485nm (SpectraMax M5, Molecular devices).

\section{Enzyme-linked Immunosorbent assay for secreted IL-1 $\beta$ and IL-18}

The primary cultured neurons were seeded into 6 -well plate at $5 \times 10^{5}$ per well. The neuronal cultures were treated with oligomer $A \beta 42$ at $100 \mathrm{nM}$ for $24 \mathrm{~h}$, and then infected with Ad-Dicer1-T2A:EGFP or vehicle virus Ad-EGFP at $2 \times 10^{8} \mathrm{vg} / \mathrm{mL}$ per well for $48 \mathrm{~h}$. The culture medium were collected and examined by IL-1 $\beta$ or IL-18 enzyme-linked immunosorbent assay kit following manufacturer's instruction (BOSTER Biological Technology Co. Ltd, Wuhan, China).

\section{Neurite outgrowth assay}

The neuronal cultures were treated by oligomer $\mathrm{A} \beta 42$ at $100 \mathrm{nM}$ for $24 \mathrm{~h}$ and then infected with Ad-EGFP or Ad-Dicer1-T2A:EGFP virus $\left(5 \times 10^{7} \mathrm{vg} / \mathrm{mL}\right)$ for $48 \mathrm{~h}$. For determination of neurite, the neurites at least two times longer than the soma diameter 
were included in the measurement (Image pro plus, Olympus Optical Co., Ltd, Tokyo, Japan). The measurement of neurite length was conducted in three independent culture preparation and the values were averaged from 150 infected neurons in either group.

\section{Western blot analysis}

The 4- or 11-month-old mice were fasted overnight, and at the next morning, mice were deeply anesthetized by isoflurane and transcardially perfused with ice-cold phosphate buffer (PBS). The hippocampi were isolated and freshly frozen in liquid nitrogen. For analyzing the neurons treated by amyloid peptide, the cytoplasmic and nuclear proteins were extracted by plasma and nuclear protein isolation kit (Cat\# P0027, Beyotime Biotechnology), respectively. For analyzing the cells for overexpressing Nrf2 plasmid, SK-N-BE (2) or N2A cells were seeded in 6-well plates at the density of $1 \times 10^{6}$ or $5 \times 10^{5}$ cells/well, respectively. Tissue or cell samples were homogenized in Super RIPA buffer composed of components in mM: 460 Tris- $\mathrm{HCl}, \mathrm{pH} 7.4,138 \mathrm{NaCl}, 1 \mathrm{EDTA}$, 2.5 NaF, 2.5 Na3VO4, 1 phenylmethanesulfonylfluoride, 1 dithiothreitol, supplemented with $0.1 \%$ Nonidet $\mathrm{P}-40$, and $1 \times$ protease/ $1 \times$ phosphatase inhibitor cocktail (Sigma, St.Louis, MO). Proteins were resolved on 12\% SDS-PAGE gels (Bio-Rad) and transferred to nitrocellulose membranes (ThermoFisher). Membranes were blocked in blocking buffer (Beyotime Biotechnology) and then incubated with primary antibody. Following wash, the membranes were then incubated with secondary antibody, and 
developed with chemiluminescence system kit (ThermoFisher). The blots were quantified using Image $\mathrm{J}$ software (National Institutes of Health, Bethesda, MD, USA).

\section{Immunofluorescence}

The 4- or 11-month-old WT, APP/PS1 non-fasted mice were deeply anesthetized by isoflurane, killed, and then transcardially perfused with ice-cold PBS followed by 4\% paraformaldehyde (PFA) in PBS. The isolated brains were postfixed in 4\% PFA overnight, then stored in PBS containing $0.01 \%$ sodium azide. Brains were mounted in agarose and $10 \mu \mathrm{m}$ coronal sections were obtained using a Leica VT1000S Vibratome. Antigen demasking was performed in $0.01 \mathrm{M}$ sodium citrate solution $\left(\mathrm{pH} \mathrm{6.0)}\right.$ at $99^{\circ} \mathrm{C}$ for 40 min. After washing the sections in PBS three times, 5 min each, at room temperature (RT), the sections were blocked using 5\% normal donkey serum in PBS containing $0.2 \%$ Triton $\mathrm{X}-100$ for $1 \mathrm{~h}$ at $\mathrm{RT}$. The sections were incubated with the rabbit anti-NeuN (1:800, Beyotime Biotechnology) or monoclonal anti-Dicer 1(1:300, SigmaAldrich) overnight at $4^{\circ} \mathrm{C}$. For mice injected with virus, the sections were incubated with anti-Dicer 1(1:300, Sigma-Aldrich) or anti-activated caspase 3 (1:300, Beyotime Biotechnology). After washing 3 times with PBS ( $3 \times 5$ min at RT), sections were incubated with secondary antibodies: Alexa Fluor 594 goat anti-rabbit or Alexa Fluor 488 goat anti-mouse IgG (1:1000, Life Technologies Corporation, Carlsbad, CA, USA) for $1 \mathrm{~h}$ at RT, then washed three times with PBS ( $3 \times 5$ min at RT). The sections were then mounted on coverslips (Citotest Labware Manufacturing Co., Ltd, Jiangsu, China) 
and the images of CA3 hippocampus were obtained with a fluorescent microscope (DMi8, Leica Biosystems).

\section{Experimental design and statistical analysis}

During Water Maze test, mice were evenly allocated to each experimental group according to the gender and ages and the tests were conducted in a blinded manner, that is, the investigator was blinded to the group allocation of the genotype or treatment during the experiment and when assessing all the results. Animal experiment sample size calculator: http://www.lasec.cuhk.edu.hk/sample-size-calculation.html was used to determine the sample sizes, assuming $40 \%$ difference in the mean and standard deviation of $20 \%$ at power 95\% and type I error 0.01 (Jiang et al., 2017). Similarly, determination of neurite outgrowth was conducted in a blinded manner. All the data acquired during experiments were included for analysis and there were no sample size differences between the beginning and end of experiments. All data are presented as means \pm SEM unless specified. Single data points were shown as overlaying dot-plot on bar-graph when sample sizes were smaller than 15, otherwise, shown as bar-graph. For parametric data, Student's t-test was used for comparing differences between two groups. In experiments with more than two groups, one-way ANOVA was performed followed by Tukey's post hoc test for comparisons among groups. For analysis of two groups of non-parametric data, the Mann-Whitney $U$-test was used for determining the differences. For Morris water maze experiment, two-way ANOVA with repeat measures followed by Tukey's post hoc test was used for analyzing 
the time spent in four quadrants, frequency to crossing platform, swimming speed and distance. Statistical analyses were performed with Graphpad Prism 7.04 software (Graphpad software, Inc. La Jolla, CA, USA). Difference was regarded as significance when $p<0.05$. 


\section{Results}

Dicer1 was reduced in the hippocampus and cortex of APPIPS1 mice and Dicer1 knockdown induced neurotoxicity

Dicer1 is reduced in the retinal pigment epithelial cells in geographic atrophy form of age-related macular degeneration(Kaneko et al., 2011) and Dicer1 knockout induces neurodegeneration (Davis et al., 2008; Shin et al., 2009). In view of these evidence, we explored Dicer1 expression in AD mice, APP/PS1. Dicer1 staining was significantly reduced in the CA3 hippocampus of APP/PS1 mice compared to WT littermate at the age of 4 months or 11 months (Figure.1A). The protein was also significantly reduced in the homogenates from the hippocampus of 4-month APP/PS1 mice compared to WT littermate $(p=0.0092, n=6$ for each genotype, Mann-Whitney $U$-test $)$ and this reduction became severe at the age of 11 months $(p=0.0002, n=6$ for each genotype, MannWhitney U-test ) (Figures.1B and 1C). Similarly, Dicer1 was significantly reduced in the homogenates from the parietal lobe of APP/PS1 mice at the age of 6 months when compared to WT littermate $(p=0.022, n=6$ for each genotype, Mann-Whitney $U$ test)(Supplemental Figure.1). Nrf2 is a master regulator of anti-oxidation genes regulating redox homeostasis(Rada et al., 2012); Dicer1 depletion induces cytotoxicity via oxidative stress(Kaneko et al., 2011). Thus, we simultaneously detected the protein levels of Nrf2 and activated caspase3 in the homogenates from APP/PS1 hippocampi. Similarly, Nrf2 protein levels in AD mice were significantly reduced compared to WT littermate at the age of 4 months ( $p=0.0001$, Mann-Whitney $U$-test) and 11 months ( $p=0.0038$, Mann-Whitney U-test), respectively (Figures. 1B and 1D). Activated caspase3 in AD mice was significantly increased compared to WT littermate at the age 
of 4 months ( $p=0.0098$, Mann-Whitney $U$-test) and 11 months ( $p=0.0008$, MannWhitney $U$-test), respectively (Figures. 1B and 1E).

Dicer1 depletion induced cytotoxicity involving the roles of ROS and inflammasome in the RPE cells (Tarallo et al., 2012). Thus, we explored the relevant effects by knocking down Dicer1(Dicer1 siRNA), the effect compared to the cells transfected with negative control scrambled siRNA (NC siRNA) in the primary murine cultures of cortical neurons $(\mathrm{CNs}, \mathrm{t}(2)=19.86, \mathrm{p}=0.0025$, paired $t$-test) or hippocampal neurons (HNs, $t(2)=4.606, p=0.044$, paired $t$-test) (Figure. 2A). As expected, Dicer1 knockdown by its specific siRNA induced ROS production in $\mathrm{CNs}(\mathrm{t}(7)=9.539, \mathrm{p}=0.00001$, paired $t$-test) or in HNs $(\mathrm{t}(7)=21.77, \mathrm{p}=0.00001$, paired $t$-test) compared to NC siRNA (Figure. 2B). Similarly, Dicer1 knockdown reduced mitochondrial membrane potential indicating by JC-1 staining in CNs (t(2)=5.085, $\mathrm{p}=0.0369$, paired $t$-test) or in $\mathrm{HNs}(\mathrm{t}(2)=4.853$, $\mathrm{p}=0.0399$, paired $t$-test) (Figures. 2C and 2D). Under expectation, Dicer1 knockdown increased the contents of interleukin-1 $\beta(\mathrm{IL}-1 \beta)$ in $\mathrm{CNs}(\mathrm{t}(2)=4.978, \mathrm{p}=0.0381$, paired $t$ test) or in HNs (t(2)=4.907, $\mathrm{p}=0.0391$, paired $t$-test) (Figure. 2E) and the production of IL-18 in CNs $(\mathrm{t}(2)=5.361, \mathrm{p}=0.0331$, paired $t$-test)or in $\mathrm{HNs}(\mathrm{t}(2)=11.86, \mathrm{p}=0.0070$, paired $t$-test ) (Figure. 2F), indicating inflammasome activation. We further explored the effect of Dicer1 knockdown on neuronal survival and its related signaling. Under the condition of Dicer1 knockdown, neuronal survival was compromised in CNs $(\mathrm{t}(2)=4.863$, $\mathrm{p}=0.0028$, paired $t$-test) or in $\mathrm{HNs}(\mathrm{t}(2)=12.1, \mathrm{p}=0.0012$, paired $t$-test) (Figure. $2 \mathrm{G})$ and the protein levels of activated caspase 3 were also increased in the $\mathrm{CNs}(\mathrm{t}(2)=11.09$, $\mathrm{p}=0.00080$, paired $t$-test) or HNs $(\mathrm{t}(2)=6.965, \mathrm{p}=0.0020$, paired $t$-test) comparing to the effects of NC siRNA (Figures. $2 \mathrm{H}$ and $2 \mathrm{l}$ ). 


\section{A 42 oligomer decreased Dicer1 and Nrf2}

As above (Figure.1), we found that Dicer1 was reduced in APP/PS1 mice which begin $A \beta$ deposition by six months, with extensive $A \beta$ plaque deposits in the hippocampus and cortex by nine months (Jankowsky et al., 2004). Thus, we investigated the effect of $A \beta 42$ on Dicer1 expression with primary murine cortical neuronal cultures. Initially, we tested the effects of neuronal cultures subject to $A \beta 42$ oligomer treatment, which reduced Dicer1 mRNA in $\mathrm{CNs}(\mathrm{t}(2)=5.879, \mathrm{p}=0.0277$, paired $t$-test $)$ and $\mathrm{HNs}(\mathrm{t}(2)=4.936, \mathrm{p}=0.0387$, paired $t$-test $)$ and increased B1 RNA in CNs $(\mathrm{t}(2)=4.453, \mathrm{p}=0.0469$, paired $t$-test $)$ and $\mathrm{HNs}(\mathrm{t}(16)=6.177, \mathrm{p}=0.0252$, paired $t$-test $)$ (Supplemental Figure. 2). As expected, Aß42 treatment reduced Dicer1 compared to sham treatment $(\mathrm{p}=0.00001)$, which was rescued by $\mathrm{N}$-acetyl-cysteine, an antioxidant $(p=0.032)(F(2,6)=73.55, p=0.00001, A N O V A)($ Figures.3A and 3B). Simultaneously, we examined total Nrf2 and found that Nrf2 was reduced by Aß42 $(p=0.0005)$, which was rescued by addition of $\mathrm{N}$-acetyl-cysteine $(\mathrm{p}=0.0009)(\mathrm{F}(2,6)=37.95, \mathrm{p}=0.0004$, ANOVA $)$ (Figures. 3A and 3C). Since Keap1 regulates Nrf2 stability, we examined Keap1 protein levels and found that $A \beta 42$ increased Keap1 $(p=0.0014)$, which was reduced by addition of $\mathrm{N}$-acetyl-cysteine $(\mathrm{p}=0.0003)(\mathrm{F}(2,6)=42.78, \mathrm{p}=0.0003$, ANOVA) (Figures. 3A and 3D). Since Nrf2 affects anti-oxidant gene transcription via binding with AREs in nucleus (Itoh et al., 2004), we further examined the levels of nucleic or cytosolic Nrf2 under $A \beta 42$ treatment or those under simultaneous addition of $\mathrm{N}$-acetyl-cysteine. $\mathrm{A} \beta 42$ reduced either nucleic Nrf2 ( $p=0.0326)$ (Figures. 3E and 3G) or cytosolic Nrf2 $(p=0.0076)$ (Figures. 3F and 3H), which was rescued by addition of $\mathrm{N}$-acetyl-cysteine $(\mathrm{p}=0.00001$ 
for nucleic $)(F(2,6)=73.82, p=0.00001$, ANOVA $)$ or $(p=0.0419$ for cytosolic $)(F(2,6)=11.73$, $\mathrm{p}=0.0084$, ANOVA), respectively (Figures. $3 \mathrm{G}$ and $3 \mathrm{H}$ ).

\section{Nrf2 regulated Dicer1 expression}

Nrf2 is a master regulator of transcription of anti-oxidant genes. We hypothesized that Nrf2 may regulate Dicer1 expression. To reach the purpose, we initially tested Dicer1 expression in SK-N-BE(2) cells with overexpression of Nrf2 compared to the cells transfected with vehicle plasmid $(p=0.0017)(F(2,6)=30.82, p=0.0007$, ANOVA)(Figures. 4A and 4C). Indeed, overexpression of Nrf2 significantly increased Dicer1 expression when compared to the cells transfected with vehicle plasmid $(p=0.0021)(F(2,6)=21.96, p=0.0017, A N O V A)$ (Figures. 4A and 4B). To further consolidate the regulation of Dicer1 by Nrf2, we further examined Dicer1 expression in Neuro-2a cells with overexpression of $\mathrm{Nrf2}(\mathrm{t}(2)=6.473, \mathrm{p}=0.024$, paired $\mathrm{t}$-test) (Figures.4D and 4F), which significantly increased Dicer1 expression when compared to the cells transfected with vehicle plasmid $(\mathrm{t}(2)=10.86, \mathrm{p}=0.0004$, paired $t$-test)(Figures. $4 \mathrm{D}$ and $4 \mathrm{E}$ ). We further scanned the promoter of Dicer1 with software (promoter 2.0 prediction server) and found three AREs motif in the proximal promoter region around transcription start site (tss). We cloned the ARE1-ARE3 into PGL6-basic plasmid containing firefly luciferase-coding sequence (-Luc), respectively, and also cloned the promoters containing different ARE motif into PGL6-basic plasmid, respectively $(F(17,54)=27.87, p=0.00001$, ANOVA $)$ (Figure. 4G). Transfection of Nrf2-expressing 
plasmid (pCMV Nrf2) increased the ratios between firefly and renilla in constructs containing ARE1(p=0.000219) or ARE3 motif ( $p=0.043)$ when compared to the cells transfected with vehicle plasmid, pCMV3. When compared the constructs containing different AREs under transfection with pCMV Nrf2, we found that the ratios of firefly/renilla from ARE1 constructs were significantly from the construct ARE2 $(p=0.000825)$, ARE3 $(p=0.0093)$, and PGL6-basic $(p=0.00041)$, respectively.

Transfection of pCMV Nrf2 increased firefly/renilla in promoter $1(p=0.00047)$, promoter $2(p=0.000135)$, promoter $3(p=0.000114)$, and mutant promoter $1(p=0.048)$ when compared to the cells transfected with pCMV3. When compared different promoter constructs under transfection with pCMV Nrf2, we found that the ratios of firefly/renilla from promoter 1 were significantly from promoter $0(p=0.000361)$ and mutant promoter $1(p=0.009)$ but not different with promoter $2(p=0.194)$ or promoter $3(p=0.254)$. Basing on these data, we projected that ARE1 motif was critical for Nrf2 regulation of Dicer1 gene transcription and expression.

\section{Overexpression of Dicer1 recovered neurotoxicity by $A \beta 42$ oligomer}

Since we have demonstrated that Dicer1 depletion compromised cell survival (Figure 2), we wondered whether overexpression of Dicer1 mitigated A 4 42-induced neurotoxicity. A $A 42$ oligomer treatment reduced the protein levels of Dicer1 $(p=0.033)$ compared to sham treatment, which was rescued by infection with Ad-Dicer1T2A:EGFP virus compared to infection with vehicle virus, Ad-EGFP $(p=0.0074)$ 
$(F(2,6)=3.15, p=0.0213, A N O V A)$ (Figures. 5A and 5B). A $A 42$ oligomer treatment increased activated caspase 3 compared to sham treatment $(p=0.0061)$, which was rescued by infection with Ad-Dicer1-T2A:EGFP virus compared to infection with AdEGFP $(p=0.023)(F(2,6)=2.05, p=0.0438, A N O V A)$ (Figures. 5A and 5C). Under expectation, $A \beta 42$ oligomer decreased neuronal survival ( $p=0.00067)$, which was significantly rescued by infection with Ad-Dicer1-T2A:EGFP virus $(p=0.047)(F(2,6)=2.46$, $\mathrm{p}=0.0362$, ANOVA) (Figure. 5D). Notably, Aß42 oligomer increased the secretion of IL$1 \beta$ (Figure. 5E) $(p=0.023)$ or IL-18 (Figure. 5F) $(p=0.019)$, which was decreased by infection with Ad-Dicer1-T2A:EGFP virus $(F(2,6)=3.18, p=0.0263$, ANOVA) (Figure. 5E, $\mathrm{p}=0.005$ for IL-1 $\beta$ secretion) and $(\mathrm{F}(2,6)=2.72, \mathrm{p}=0.0328$, ANOVA)(Figure. $5 \mathrm{~F}, \mathrm{p}=0.04$ for IL-18 secretion). Dicer1 knockdown disrupted neurite in primary cortical neurons $(\mathrm{t}(16)=2.51, \mathrm{p}=0.011$, paired $t$-test) (Supplemental Fig3), an early pathological change in AD. Similarly, $A \beta 42$ oligomer treatment trimmed neurite $(p=0.025)$, which was significantly recovered by overexpression of Ad-Dicer1-T2A:EGFP virus $(p=0.047)(F(2$, 447) $=3.24, p=0.0285$, ANOVA) (Figures. $5 \mathrm{G}$ and $5 \mathrm{H}$ ).

\section{Overexpression of Dicer1 improved spatial learning in APP/PS1 mice}

We further explored the effect of overexpression of Dicer1 in APP/PS1mice. Consistent with previous observation (Figure. 1A), Dicer1 staining was decreased in CA3 hippocampus compared to WT littermate at the age of 3.5 months; injection of AdDicer1:T2A:EGFP significantly increased Dicer1 staining in CA3 hippocampus in 
APP/PS1 mice (Figure. 6A). CA3 hippocampi of APP/PS1mice contained stronger staining for activated caspase 3 compared to those of WT littermate, which was reduced by injection of Ad-Dicer1:T2A:EGFP virus (Figure. 6B). In parallel experiment, we isolated CA3 hippocampi and subjected the homogenates to western blot against Dicer1 and caspase 3. Consistent with the immunofluorescence, there was lower level of Dicer1 in APP/ PS1mice than WT ( $p=0.0217)$ which was recovered by injection of AdDicer1:T2A:EGFP ( $p=0.0093)$ ( $p=0.00236$, Kruskal-Wallis test) (Figure. 6D); there was higher level of activated caspase 3 in APP/PS1mice than WT ( $p=0.00681)$ which was reduced by injection of Ad-Dicer1:T2A:EGFP ( $p=0.0067)(p=0.0036$, Kruskal-Wallis test) (Figure. 6E). We further investigated whether overexpression of Dicer1 had effect on spatial learning and thus examined behavior with Morris Water Maze. During the training period on days 1-6 after virus injection, the latency to reach platform for APP/PS1 mice was significantly reversed by injection with Ad-Dicer1:T2A:EGFP virus compared to injection with Ad1-EGFP virus $(p=0.008)$ on day $5(F(2,42)=9.042$ $p=0.0057$, ANOVA $)$, and $(p=0.00001)$ on day $6(F(2,42)=13.12, p=0.0025$, ANOVA $)$ (Figure. 7A). When combining timepoint and treatment, the latency was significantly reversed by injection with Ad1-Dicer1:T2A:EGFP virus $(p=0.0001)(F$ day $(5,174)=36.44$, $p=0.00001 ;$ Ftreatment $(2,174)=2.045, p=0.0241 ;$ Finteraction $(75,174)=2.3, p=0.0148$, two-way ANOVA) (Figure. 7A). On day 7 after a 60-s probe test for searching platform, we monitored animal's swimming pattern, distance, speed, and the amount of time spent in each of the four quadrants. Without difference on swimming distance (F (2, $42)=1.19, p=0.3141$, ANOVA) (Figure. 7D) and swimming speed $(F(2,42)=1.49$, $\mathrm{p}=0.0948$, ANOVA) (Figure. 7E), APP/PS1mice spent less time in target quadrant than 
WT mice ( $p=0.0023$ ), which was reversed by injection with Ad-Dicer1:T2A:EGFP virus $(p=0.0087)\left(F(2,42)=5.43 P=0.0092 ; F_{\text {quadrant }}(3,116)=4.576, p=0.1122 ; F_{\text {treatment }}\right.$ $(2,116)=0.1032, p=1 ; F_{\text {interaction }}(45,116)=1.334 . p=0.0046$, two-way ANOVA) (Figure 7B). Similarly, the AD mice crossed the platform less frequently than WT mice $(p=0.039)$, but the chance was increased by injection with Ad-Dicer1:T2A:EGFP virus $(p=0.043)(F(2,42)=4.967, p=0.0116$, ANOVA $)($ Figure. $7 C)$. 


\section{Discussion}

In this study, we indicated that Dicer1 was reduced in the hippocampus and cortex of APP/PS1 mice in vivo and Dicer1 was reduced by Aß42 oligomer in vitro. Dicer1 depletion led to neurite deficit and apoptosis via oxidative injury-mediated inflammasome activation such as increased ROS production and activated caspase3, damaged mitochondrial transmembrane potential, and IL-1 $\beta$ and IL-18 secretion. We further indicated that Nrf2 increased Dicer1 expression which was linked with Keap1Nrf2-ARE signaling pathway. Overexpression of Dicer1 increased neuronal survival and recovered neurite deficit by $\mathrm{A} \beta 42$ oligomer in vitro, and in vivo Dicer1 overexpression in CA3 hippocampus improved spatial learning in APP/PS1 mice. Altogether, we reveal a novel role of Dicer1 in AD and a novel regulation of Dicer1. In our study, reduction of Dicer1 was detected as early as around 4 months in APP/PS1 mice which begin $A \beta$ deposition at the age of 6 months (Jankowsky et al., 2004). Furthermore, knockdown of Dicer1 in cultured neurons induced neurite deficit before soma degeneration, and intrahippocampal injection of Dicer1-expressing virus improved spatial learning. These evidence suggest that reduction of Dicer1 is an early event in $A D$ and targeting Dicer1 may provide a new strategy for AD therapy with emphasis on the early stage of this disorder.

Oxidative stress is thought to be central in the pathogenesis of $A D$, for example, oxidative stress exacerbates insulin resistance in AD brain (De Felice et al., 2014) and disrupts mitochondria (Lin and Beal, 2006), resulting in failure to produce sufficient ATP for $\mathrm{Na}^{+}-\mathrm{K}^{+}$-ATPase and $\mathrm{Na}^{+}-\mathrm{Ca}^{++}$exchanger to maintain ion homeostasis in cells (Reeves et al., 1986; Gloor, 1997; Kip and Strehler, 2007). Dicer1 depletion may be an 
internal sources contributing to oxidative stress by accumulation of Alu RNA (Tarallo et al., 2012). Intrahippocampal injection of Nrf2-expressing lentivirus improves spatial learning in APP/PS1 mice (Kanninen et al., 2009) but Nrf2 promotes cancer cell proliferation(Jaramillo and Zhang, 2013). As such, manipulation of Nrf2 expression may lead to undesired side effects although it indicates potential benefit in AD brain (Kanninen et al., 2009). By contrast, appropriate expression of Dicer1 may be an alternative strategy to oppose oxidative stress in AD brain. Dicer1 is ubiquitously expressed in the brain including in neurons, astroglia, and oligodendrocytes (Cuellar et al., 2008; Shin et al., 2009; Tao et al., 2011). Thus, Dicer1 reduction in APP/PS1 brain and secondary neurodegeneration are the combinatory effects from neurons and glia. Therefore, it is important to determine the contribution of Dicer1 reduction in cell type in AD brain for therapy purpose in the future. Nrf2 mediates Dicer1 expression via Keap1Nrf2-ARE signaling pathway, and decreased Nrf2-ARE signaling may mediate the reduction of Dicer1 in AD brain as projected from our study. Overexpression of Dicer1 may serve as a forward regulator of Nrf2, which is unclear yet.

Dicer1 is central to the processing from pre-microRNAs to mature microRNAs, which regulate neuronal survival and neuritogenesis (Im and Kenny, 2012; Carrella et al., 2015). Deletion of functional Dicer1 has been found to induce accumulation of Alu RNA and activate NLRP3 inflammasome and oxidative damages in age-related macular degeneration(Kaneko et al., 2011; Tarallo et al., 2012). Conditional ablation of Dicer1 in oligodendrocytes induces neuronal impairment via demyelination, oxidative damage, inflammatory astrocytosis and microgliosis in mice (Shin et al., 2009). There is also evidence showing that conditional knockout of Dicer1 in dopaminergic neurons of 
ventral midbrain leads to neuronal loss through inhibiting micoRNAs biosynthesis(Chmielarz et al., 2017). Similarly, blocking microRNAs biosynthesis by knocking out Dicer1 disrupts morphogenesis in the cortex and hippocampus (Davis et al., 2008). Apart from Alu RNA accumulation, microRNAs deficiency due to Dicer1 depletion may play unexpected roles in AD neurodegeneration as Alu RNA, which is of great interest to explore.

In summary, we revealed novel roles of Dicer1 in AD brain and a novel regulation of Dicer1. Our findings suggest Dicer1 may be a target in AD therapy. 
bioRxiv preprint doi: https://doi.org/10.1101/711572 t this version posted July 23,2019 . The copyright holder for this preprint (which was not certified by peer review) is the author/funder, who has granted bioRxiv a license to display the preprint in perpetuity. It is made available under aCC-BY-NC-ND 4.0 International license.

\section{Author contribution}

YW designed, conducted the experiments, analyzed data, and wrote the first draft; MLL conducted the experiments; JZ began the characterization of Dicer1 expression in APP/PS1 mice; SZW conceived of this project, analyzed data, and wrote the final manuscript. 


\section{References}

Belaidi AA, Bush Al (2016) Iron neurochemistry in Alzheimer's disease and Parkinson's disease: targets for therapeutics. J Neurochem 139 Suppl 1:179-197.

Bernstein E, Caudy AA, Hammond SM, Hannon GJ (2001) Role for a bidentate ribonuclease in the initiation step of RNA interference. Nature 409:363-366.

Carrella S, D'Agostino Y, Barbato S, Huber-Reggi SP, Salierno FG, Manfredi A, Neuhauss SC, Banfi S, Conte I (2015) miR-181a/b control the assembly of visual circuitry by regulating retinal axon specification and growth. Developmental neurobiology 75:1252-1267.

Chen ZH, Saito Y, Yoshida Y, Sekine A, Noguchi N, Niki E (2005) 4-Hydroxynonenal induces adaptive response and enhances PC12 cell tolerance primarily through induction of thioredoxin reductase 1 via activation of Nrf2. J Biol Chem 280:41921-41927.

Chmielarz P, Konovalova J, Najam SS, Alter H, Piepponen TP, Erfle H, Sonntag KC, Schutz G, Vinnikov IA, Domanskyi A (2017) Dicer and microRNAs protect adult dopamine neurons. Cell Death Dis 8:e2813.

Cuellar TL, Davis TH, Nelson PT, Loeb GB, Harfe BD, Ullian E, McManus MT (2008) Dicer loss in striatal neurons produces behavioral and neuroanatomical phenotypes in the absence of neurodegeneration. Proc Natl Acad Sci U S A 105:5614-5619.

Davis TH, Cuellar TL, Koch SM, Barker AJ, Harfe BD, McManus MT, Ullian EM (2008) Conditional loss of Dicer disrupts cellular and tissue morphogenesis in the cortex and hippocampus. J Neurosci 28:4322-4330.

De Felice FG, Lourenco MV, Ferreira ST (2014) How does brain insulin resistance develop in Alzheimer's disease? Alzheimer's \& dementia : the journal of the Alzheimer's Association 10:S26-32.

Gloor SM (1997) Relevance of Na,K-ATPase to local extracellular potassium homeostasis and modulation of synaptic transmission. FEBS Lett 412:1-4.

Halliwell B (2006) Oxidative stress and neurodegeneration: where are we now? J Neurochem 97:1634-1658.

Hardy J, Selkoe DJ (2002) The amyloid hypothesis of Alzheimer's disease: progress and problems on the road to therapeutics. Science 297:353-356.

Heneka MT, Kummer MP, Stutz A, Delekate A, Schwartz S, Vieira-Saecker A, Griep A, Axt D, Remus A, Tzeng TC, Gelpi E, Halle A, Korte M, Latz E, Golenbock DT (2013) NLRP3 is activated in Alzheimer's disease and contributes to pathology in APP/PS1 mice. Nature 493:674-678.

Im HI, Kenny PJ (2012) MicroRNAs in neuronal function and dysfunction. Trends in neurosciences 35:325-334.

Itoh K, Tong KI, Yamamoto M (2004) Molecular mechanism activating Nrf2-Keap1 pathway in regulation of adaptive response to electrophiles. Free Radic Biol Med 36:1208-1213.

Jankowsky JL, Fadale DJ, Anderson J, Xu GM, Gonzales V, Jenkins NA, Copeland NG, Lee MK, Younkin LH, Wagner SL, Younkin SG, Borchelt DR (2004) Mutant presenilins specifically elevate the levels of the 42 residue beta-amyloid peptide 
in vivo: evidence for augmentation of a 42-specific gamma secretase. Hum Mol Genet 13:159-170.

Jaramillo MC, Zhang DD (2013) The emerging role of the Nrf2-Keap1 signaling pathway in cancer. Genes Dev 27:2179-2191.

Jiang H, Wu M, Liu Y, Song L, Li S, Wang X, Zhang YF, Fang J, Wu S (2017) Serine racemase deficiency attenuates choroidal neovascularization and reduces nitric oxide and VEGF levels by retinal pigment epithelial cells. J Neurochem 143:375388.

Kaneko H et al. (2011) DICER1 deficit induces Alu RNA toxicity in age-related macular degeneration. Nature 471:325-330.

Kanninen K, Heikkinen R, Malm T, Rolova T, Kuhmonen S, Leinonen H, Yla-Herttuala S, Tanila H, Levonen AL, Koistinaho M, Koistinaho J (2009) Intrahippocampal injection of a lentiviral vector expressing Nrf2 improves spatial learning in a mouse model of Alzheimer's disease. Proc Natl Acad Sci U S A 106:1650516510.

Keller JN, Germeyer A, Begley JG, Mattson MP (1997) 17Beta-estradiol attenuates oxidative impairment of synaptic $\mathrm{Na}+\mathrm{K}+-\mathrm{ATPase}$ activity, glucose transport, and glutamate transport induced by amyloid beta-peptide and iron. J Neurosci Res 50:522-530.

Kip SN, Strehler EE (2007) Rapid downregulation of NCX and PMCA in hippocampal neurons following H2O2 oxidative stress. Ann N Y Acad Sci 1099:436-439.

Kobayashi A, Kang MI, Okawa H, Ohtsuji M, Zenke Y, Chiba T, Igarashi K, Yamamoto M (2004) Oxidative stress sensor Keap1 functions as an adaptor for Cul3-based E3 ligase to regulate proteasomal degradation of Nrf2. Mol Cell Biol 24:71307139.

Lin MT, Beal MF (2006) Mitochondrial dysfunction and oxidative stress in neurodegenerative diseases. Nature 443:787-795.

Mark RJ, Hensley K, Butterfield DA, Mattson MP (1995) Amyloid beta-peptide impairs ion-motive ATPase activities: evidence for a role in loss of neuronal $\mathrm{Ca2+}$ homeostasis and cell death. J Neurosci 15:6239-6249.

Mark RJ, Pang Z, Geddes JW, Uchida K, Mattson MP (1997) Amyloid beta-peptide impairs glucose transport in hippocampal and cortical neurons: involvement of membrane lipid peroxidation. J Neurosci 17:1046-1054.

Markesbery WR (1997) Oxidative stress hypothesis in Alzheimer's disease. Free Radic Biol Med 23:134-147.

Mattson MP (2004) Pathways towards and away from Alzheimer's disease. Nature 430:631-639.

McWalter GK, Higgins LG, McLellan LI, Henderson CJ, Song L, Thornalley PJ, Itoh K, Yamamoto M, Hayes JD (2004) Transcription factor Nrf2 is essential for induction of $\mathrm{NAD}(\mathrm{P}) \mathrm{H}$ :quinone oxidoreductase 1, glutathione $\mathrm{S}$-transferases, and glutamate cysteine ligase by broccoli seeds and isothiocyanates. The Journal of nutrition 134:3499S-3506S.

Rada P, Rojo Al, Evrard-Todeschi N, Innamorato NG, Cotte A, Jaworski T, TobonVelasco JC, Devijver H, Garcia-Mayoral MF, Van Leuven F, Hayes JD, Bertho G, Cuadrado A (2012) Structural and functional characterization of Nrf2 degradation by the glycogen synthase kinase 3/beta-TrCP axis. Mol Cell Biol 32:3486-3499. 
Reeves JP, Bailey CA, Hale CC (1986) Redox modification of sodium-calcium exchange activity in cardiac sarcolemmal vesicles. J Biol Chem 261:4948-4955.

Rushworth JV, Griffiths HH, Watt NT, Hooper NM (2013) Prion protein-mediated toxicity of amyloid-beta oligomers requires lipid rafts and the transmembrane LRP1. J Biol Chem 288:8935-8951.

Shin D, Shin JY, McManus MT, Ptacek LJ, Fu YH (2009) Dicer ablation in oligodendrocytes provokes neuronal impairment in mice. Ann Neurol 66:843-857.

Stockwell BR et al. (2017) Ferroptosis: A Regulated Cell Death Nexus Linking Metabolism, Redox Biology, and Disease. Cell 171:273-285.

Tao J, Wu H, Lin Q, Wei W, Lu XH, Cantle JP, Ao Y, Olsen RW, Yang XW, Mody I, Sofroniew MV, Sun YE (2011) Deletion of astroglial Dicer causes non-cellautonomous neuronal dysfunction and degeneration. J Neurosci 31:8306-8319.

Tarallo V et al. (2012) DICER1 loss and Alu RNA induce age-related macular degeneration via the NLRP3 inflammasome and MyD88. Cell 149:847-859. 


\section{Figure legends}

A

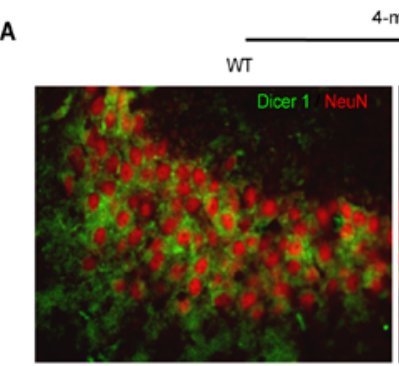

B
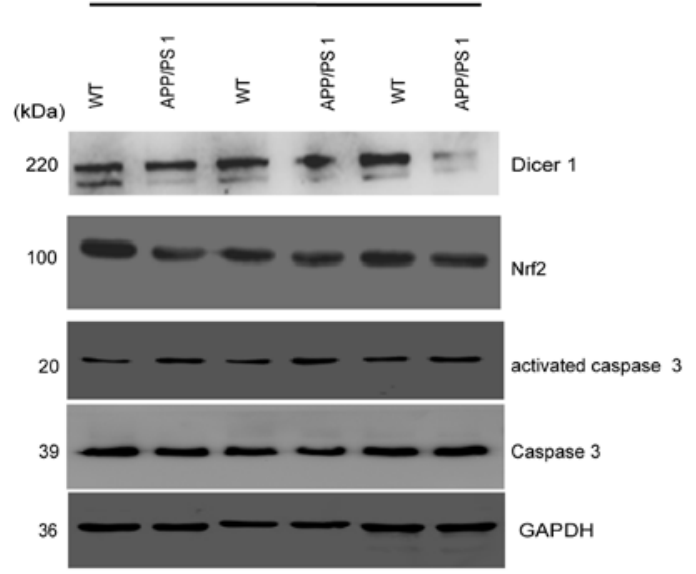

C

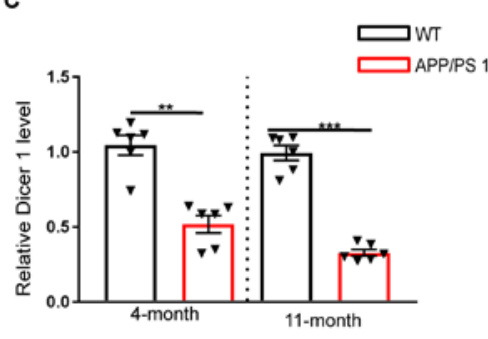

4-month

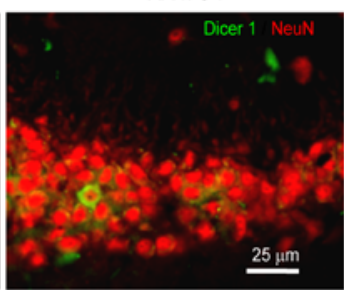

$25 \mu \mathrm{m}$

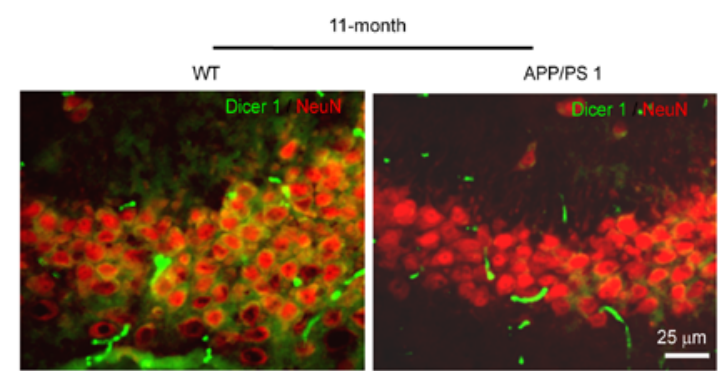

11-month
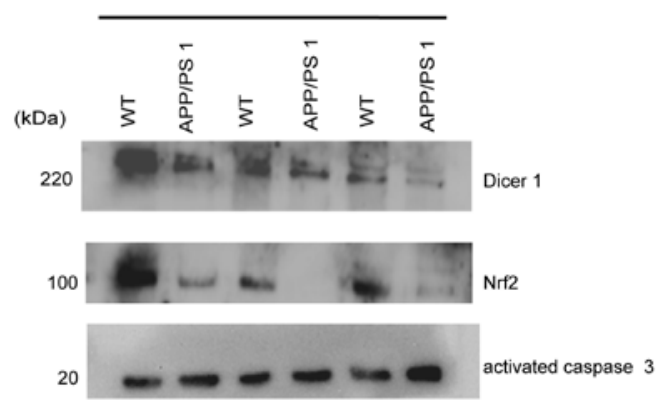

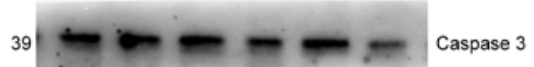

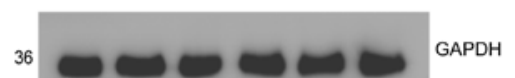

E

D

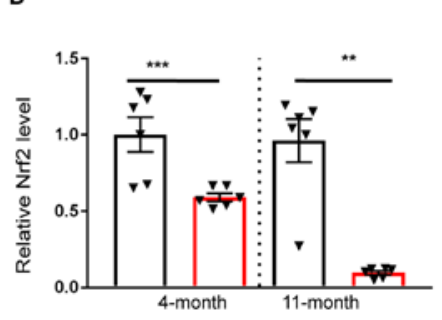

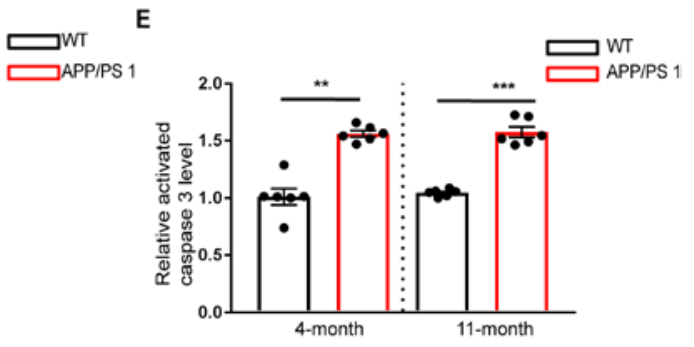

Figure 1. Reduction of Dicer 1, decreased Nrf2 and increased activated caspase-3 in the hippocampus of APP/PS1 mice. (A) APP/PS1 brains were processed into sections which were doubly stained for Dicer1(1:300) (green) or NeuN (1:800) (red). The images were typical staining of CA3 hippocampus ( $n=3$ mice per genotype). Bar, $25 \mu \mathrm{m}$ for all panels. (B) The hippocampi at the age of 4-/11 months were isolated from the brain, homogenized, and subjected to centrifugation at $12,000 \mathrm{Xg}$ for $10 \mathrm{~min}$. The supernatants were collected and subject to examination of Dicer1(1:600), Nrf2 (1:600), 
caspase-3 (1:500), and activated caspase-3 (1:600) by western blot. GAPDH (1:1500) was used as a loading control. The protein levels of Dicer1 and Nrf2 in the hippocampal tissues were reduced in APP/PS1 mice $(n=6)$ compared to WT littermate $(n=6)$, respectively, at the age of 4 months (Left panel) or at the age of 11 months (right panel). By contrast, the protein levels of activated caspase-3 were increased in APP/PS1 mice ( $n=6)$ compared to WT $(n=6)$ at the age of 4-/11 months. (C) The optical densities of Dicer1 relative to GAPDH were normalized to WT at the age of 4-/11 months (MannWhitney $U$ test, ${ }^{\star \star} \mathrm{p}=0.0092$ in $4-$ month; ${ }^{* \star} \mathrm{p}=0.0002$ in 11-month, $\mathrm{n}=6$ per genotype). (D), Nrf2 relative to GAPDH were normalized to WT (Mann-Whitney U test, ${ }^{* \star *} \mathrm{p}=0.0001$ in 4-month, ${ }^{* *} p=0.0038$ in 11-month, $n=6$ per genotype). (E) Activated caspase 3 relative to GAPDH were normalized to WT (Mann-Whitney $U$ test, ${ }^{* \star} p=0.0098$ in 4 month, ${ }^{* \star} \mathrm{p}=0.0008$ in 11-month, $\mathrm{n}=6$ per genotype), respectively. 
A

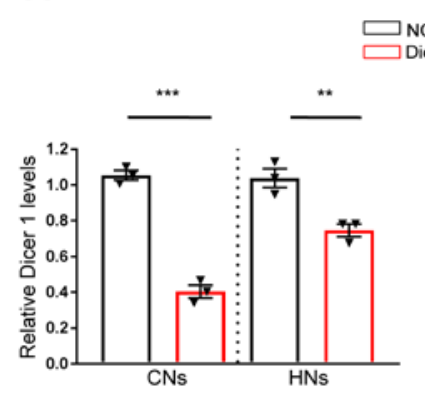

D

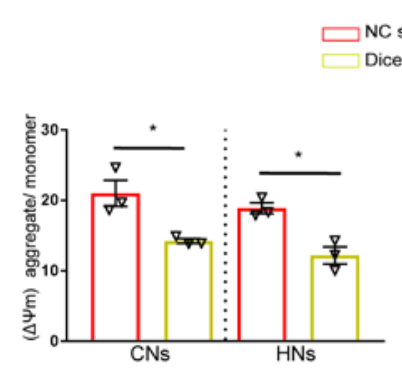

G

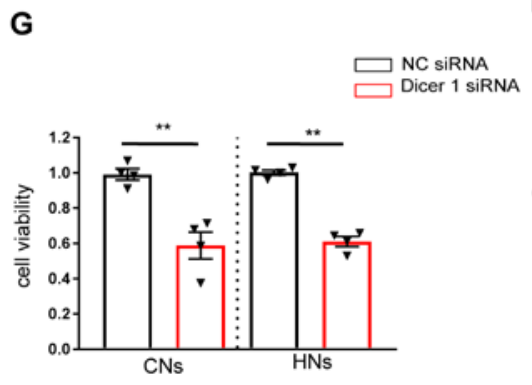

E
B
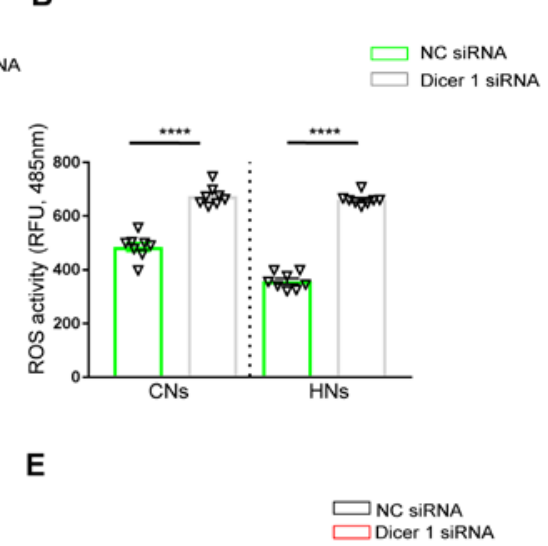

C NC siRnA
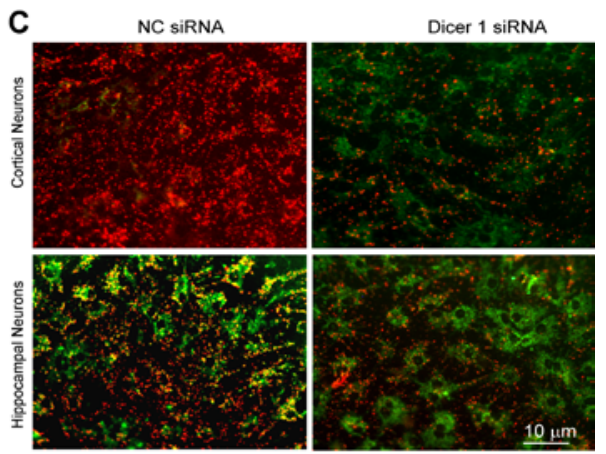

F
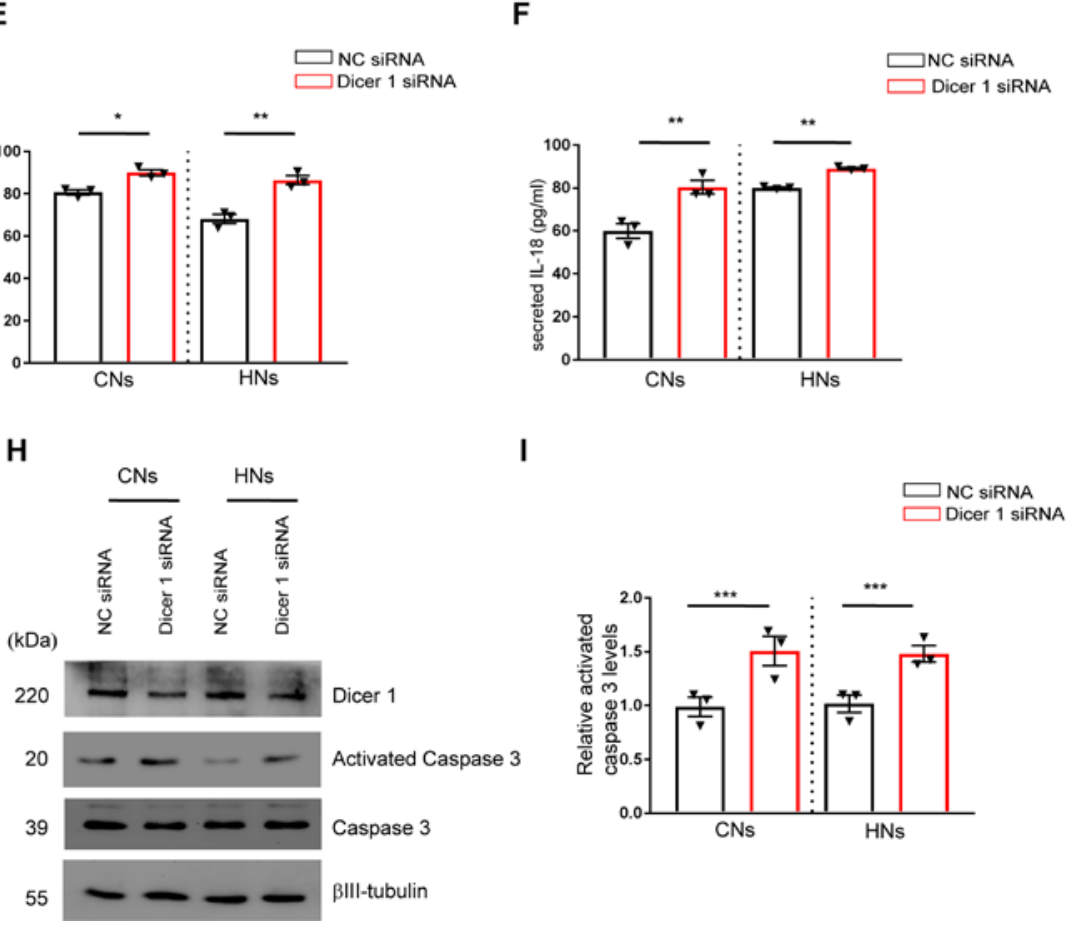

I

H
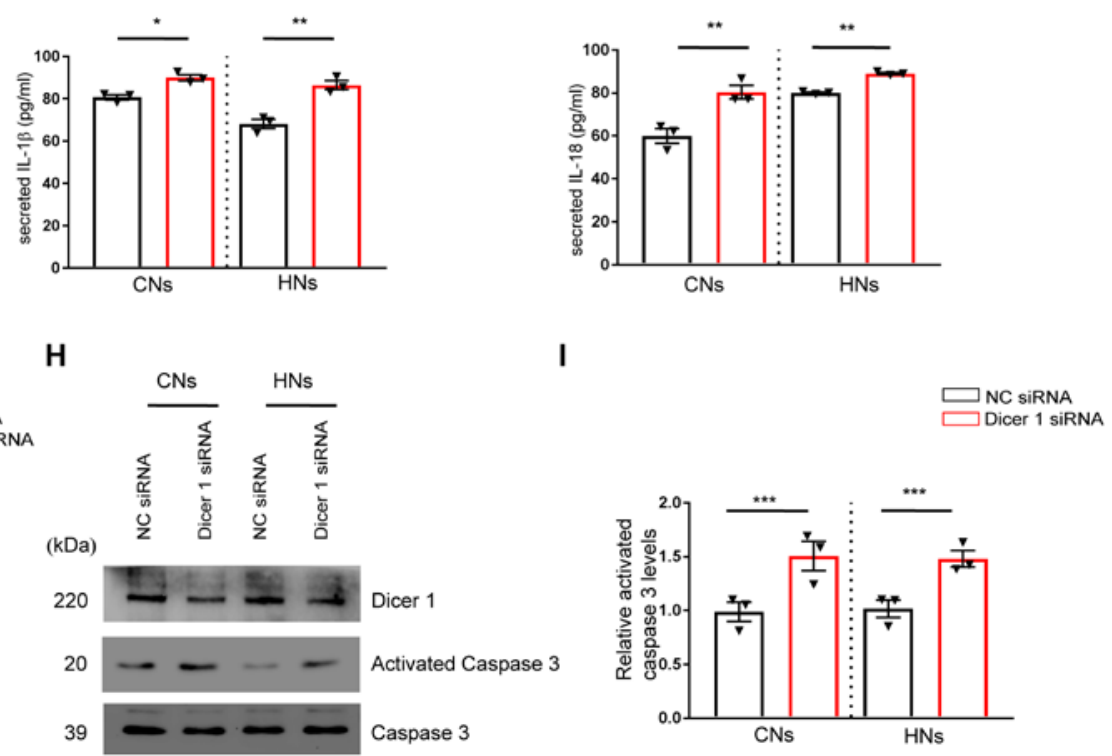

Figure 2. knocking down Dicer 1 induced production of reactive oxygen species (ROS), reduced mitochondrial membrane potential, enhanced secretion of IL-1 $\beta$, IL-18, and apoptosis in primary cultured neurons. (A) Primary murine cortical neurons (CNs) and hippocampal neurons (HNs) in 6-well plates were transfected with Dicer1 siRNA (50 pM) or control siRNA duplex (50 pM) per well by lipofectamine 2000 for $12 \mathrm{~h}$ in DMEM/F12 plus 2\% B27. The medium was replaced with fresh neurobasal supplemented with 2\% B27 and continued to be cultured for $36 \mathrm{~h}$. The effects of knockdown by Dicer1 siRNA were normalized to those of NC siRNA and averaged from 
three independent culture preparation. Student's $t$-test was used to compare the differences, ${ }^{*} \mathrm{p}=0.0025$ for comparison in $\mathrm{CNs}$ and ${ }^{*} \mathrm{p}=0.044$ for comparison in HNs for

Dicer 1. (B) The ROS levels in CNs or HNs were measured by incubating 2',7'dichlorodihydrofluorescein diacetate for $20 \mathrm{~min}$ at $37^{\circ} \mathrm{C}$. The fluorescence values in relative fluorescence units (RFU) were acquired in a plate reader at $485 \mathrm{~nm}$ and were averaged from four independent culture preparation with duplicate cultures in each preparation. Student's $t$-test was used to compare the differences of ROS production in neurons between transfection with Dicer1 siRNA and control siRNA. ${ }^{\star \star * *} p=0.00001$ in CNs, ${ }^{* \star *} \mathrm{p}=0.00001$ in HNs. (C) Representative images of JC-1 staining in CNs and HNs subject to Dicer1 knockdown as above, Bar $10 \mu \mathrm{m}$ for all panels. (D) Mitochondrial membrane potential $\left(\Delta \Psi_{\mathrm{m}}\right)$ of $\mathrm{CNs}$ and HNs subject to Dicer1 knockdown. The neurons were subject to Dicer1 knockdown thereof and mitochondria (30 $\mu \mathrm{g})$ were isolated and stained with $\mathrm{JC}-1$. The values of mitochondrial membrane potential $\left(\Delta \Psi_{\mathrm{m}}\right)$ were indicated by the ratios between aggregated/monomer RFU and averaged from three independent culture preparations. Student's $t$-test was used to compare the differences, ${ }^{*} p=0.0369$ in CNs, ${ }^{*} p=0.0399$ in HNs. (E, F) secreted IL-1 $\beta$ and IL-18 in CNs and HNs subject to Dicer1 knockdown. The CNs or HNs were subjected to Dicer1 knockdown as above. The culture media $(100 \mu \mathrm{L})$ from neuronal culture subjected to transfection with Dicer1 siRNA or control siRNA duplex were collected and measured by ELISA. The concentrations of IL-1 $\beta$ and IL-18 were averaged from three independent culture preparation. Student's $t$-test was used to compare the differences. ${ }^{*} \mathrm{p}=0.0381$ when comparing IL-1 $\beta$ production in $\mathrm{CNs}$ and ${ }^{*} \mathrm{p}=0.0391$ in $\mathrm{HNs}$. ${ }^{*} \mathrm{p}=0.0331$ when comparing IL-18 production in CNs and ${ }^{*} \mathrm{p}=0.007$ in HNs. (G) Viability of neurons 
subjected to Dicer1 knockdown. CNs or HNs at the density of $5 \times 10^{3}$ were transfected with Dicer 1 siRNA and NC siRNA for $12 \mathrm{~h}$ in DMEM/F12 plus 2\% B27, which was replaced with fresh neurobasal medium supplemented with $2 \%$ B27 and continued to be cultured for $36 \mathrm{~h}$, respectively. The absorption values at OD 450nm in Dicer1 siRNA group were measured and normalized to NC siRNA group. Student's $t$-test, ${ }^{\star *} \mathrm{p}=0.0028$ for comparison in CNs, ${ }^{\star \star} p=0.0012$ for comparison in HNs. (H) Representative images of Dicer 1, caspase3 and activated caspase3 in CNs and HNs subject to Dicer1 knockdown. BIII-tubulin was used as a loading control. (I) The optical densities of activated caspase-3 relative to $\beta$ III-tubulin from $\mathrm{H}$ were normalized to NC siRNA group, and averaged from three independent culture preparation. Student's $t$-test was used to compare the differences, ${ }^{\star \star \star} p=0.0008$ for comparison in $\mathrm{CNs}$ and ${ }^{\star \star \star} \mathrm{p}=0.002$ for comparison in HNs. 
A

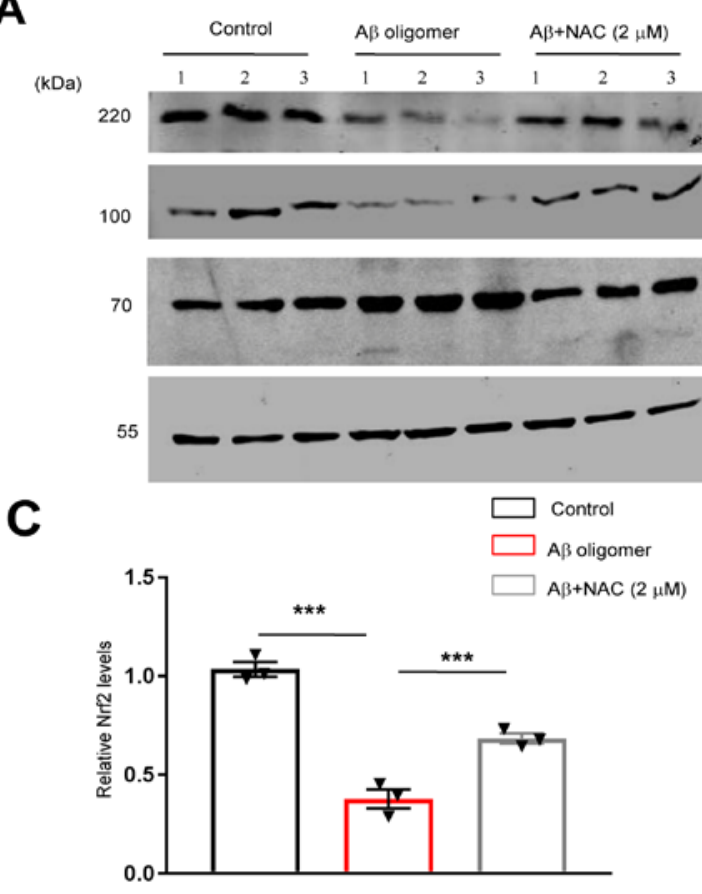

B

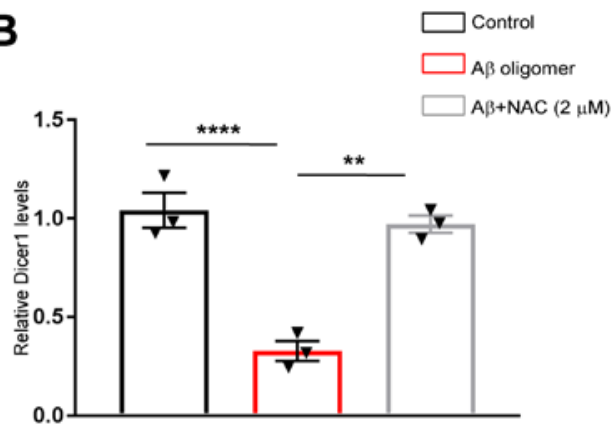

$\mathbf{E}$

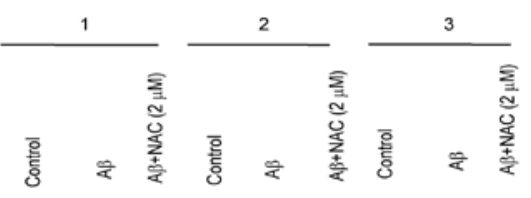

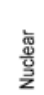

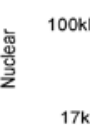

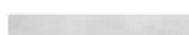

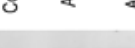

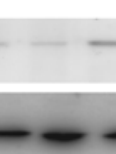

G

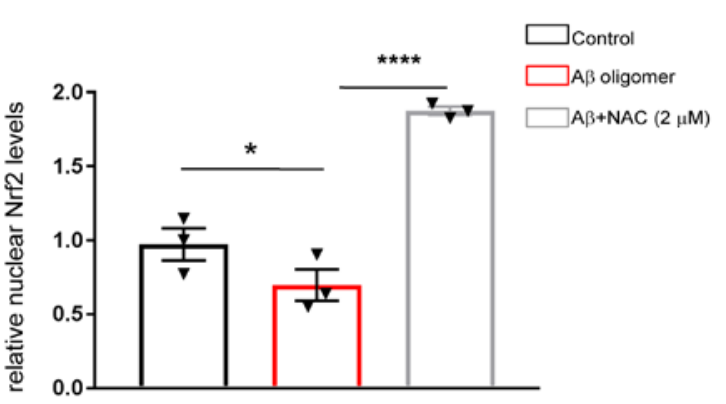

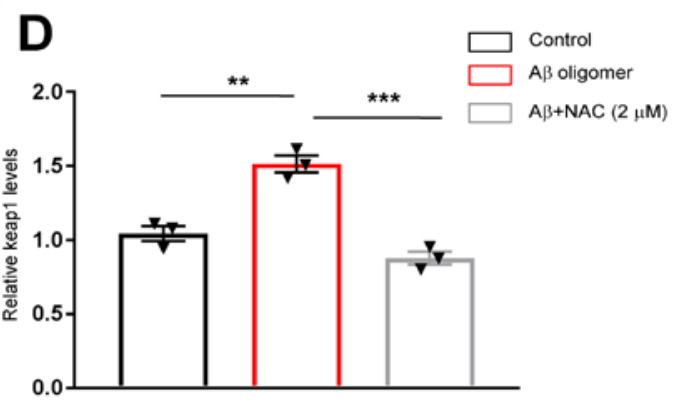

$\mathbf{F}$
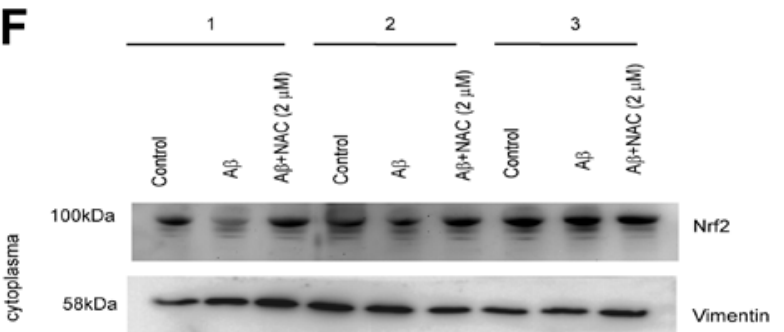

H

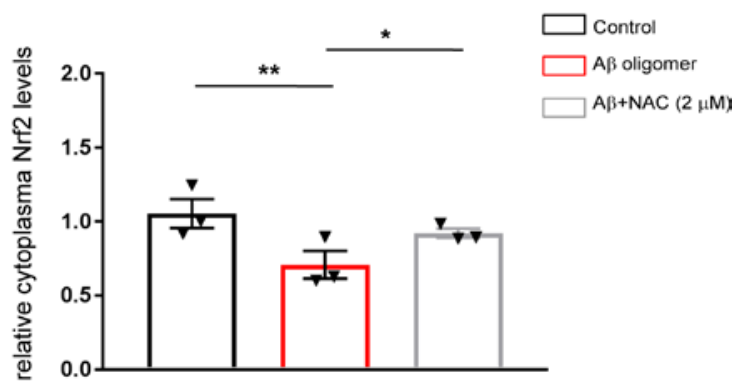

Figure 3. Aß42 oligomer reduced Dicer1 which was coupled with Nrf2 reduction, the effects rescued by NAC. The primary mouse cortical neurons were treated with Aß42 oligomer (100 nM) and antioxidant NAC $(2 \mu \mathrm{M})$ for $48 \mathrm{~h}$. (A) The neuronal cultures were harvested after the treatment, homogenized, and subject to centrifugation 
at $12,000 \times \mathrm{g}$. The supernatants were collected for western blot against Dicer 1(1:600), Nrf2 (1:600), and Keap1(1:600). BIII-tubulin (1:1000) was used as an internal loading control. (B) The optical densities of Dicer1/ßIII-tubulin were normalized to sham treatment and averaged from three independent culture preparation in A. One-way ANOVA followed by Tukey's post hoc test was used to compare the differences. Control vs. $A \beta 42$ oligomer treatment, ${ }^{\star \star \star \star} p=0.00001 ; A \beta 42$ oligomer vs. $A \beta 42$ oligomer plus NAC treatment $(A \beta+N A C),{ }^{*} p=0.032$. (C) The optical densities of Nrf2/ $\beta$ III-tubulin were normalized to sham treatment and averaged from three independent culture preparation in A. One-way ANOVA followed by Tukey's post hoc test was used to compare the differences. Control vs. $A \beta 42$ oligomer treatment, ${ }^{* \star} p=0.0005 ; A \beta 42$ oligomer vs. $A \beta+N A C,{ }^{* \star *} p=0.0009$. (D) The optical densities of Keap1/ $\beta$ III-tubulin were normalized to sham treatment and averaged from three independent culture preparation in $A$. Control vs. $A \beta 42$ oligomer, ${ }^{\star \star} p=0.0014 ; A \beta 42$ oligomer vs. $A \beta+N A C$, ${ }^{\star \star \star} \mathrm{p}=0.0003$. The neurons were treated with $\mathrm{A} \beta 42$ oligomer $(100 \mathrm{nM})$ and antioxidant NAC $(2 \mu \mathrm{M})$ for $24 \mathrm{~h}$. (E) The nucleic proteins were extracted and blotted against Nrf2 (1:600), and histone3 (1:800) served as a loading control; (F) the cytoplasmic proteins were isolated and blotted against Nrf2 (1:600), and vimentin (1:500) served as a loading control. (G) The optical densities of nucleic Nrf2 relative to histone 3 averaged from three independent culture preparation in E. One-way ANOVA followed by Tukey's post hoc test was used to compare the differences. Control vs. $A \beta 42,{ }^{\star} p=0.0326 ; A \beta 42$ vs. $A \beta+N A C,{ }^{* * *} p=0.00001 .(\mathbf{H})$ The optical densities of cytosolic Nrf2/vimentin were normalized to sham treatment and averaged from three independent culture preparation in F. One-way ANOVA followed by Tukey's post hoc test was used to compare the 
bioRxiv preprint doi: https://doi.org/10.1101/711572; this version posted July 23,2019 . The copyright holder for this preprint (which was not certified by peer review) is the author/funder, who has granted bioRxiv a license to display the preprint in perpetuity. It is made available under aCC-BY-NC-ND 4.0 International license.

differences. Control vs. $A \beta 42$ oligomer, ${ }^{*} p=0.0076 ; A \beta 42$ oligomer vs. $A \beta+N A C$, ${ }^{*} \mathrm{p}=0.0419$. 
A SK-N-BE cells

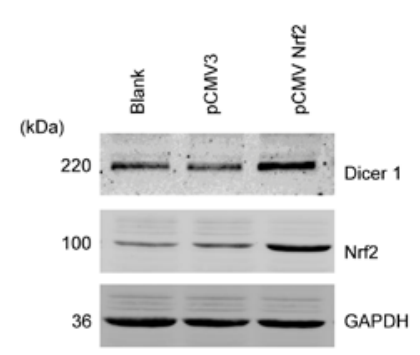

B

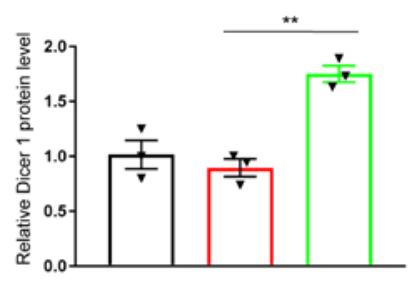

$\mathrm{E}$

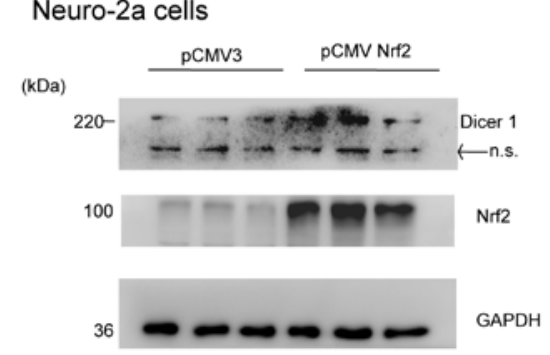

E

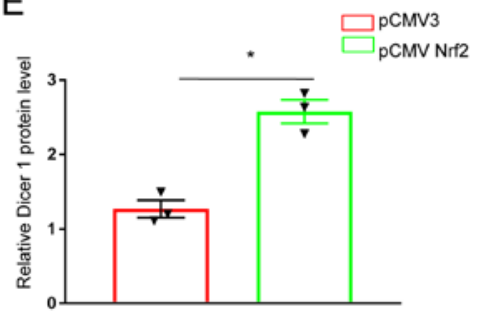

C
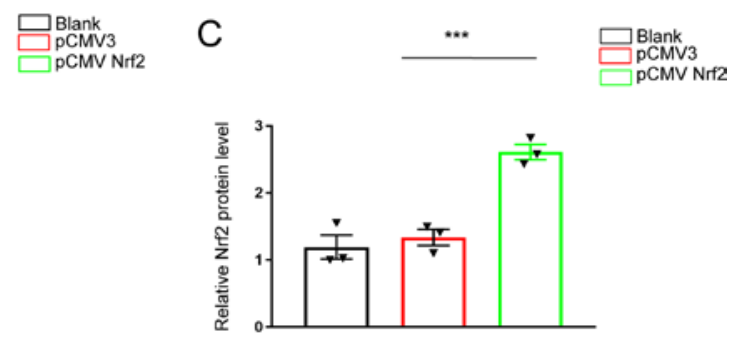

$\mathrm{F}$

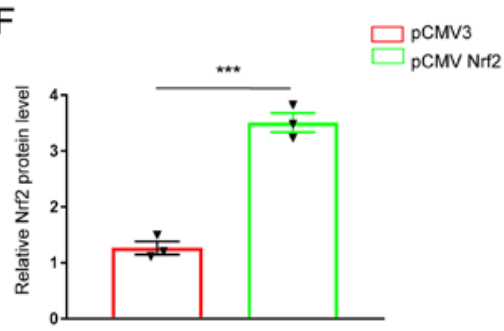

G

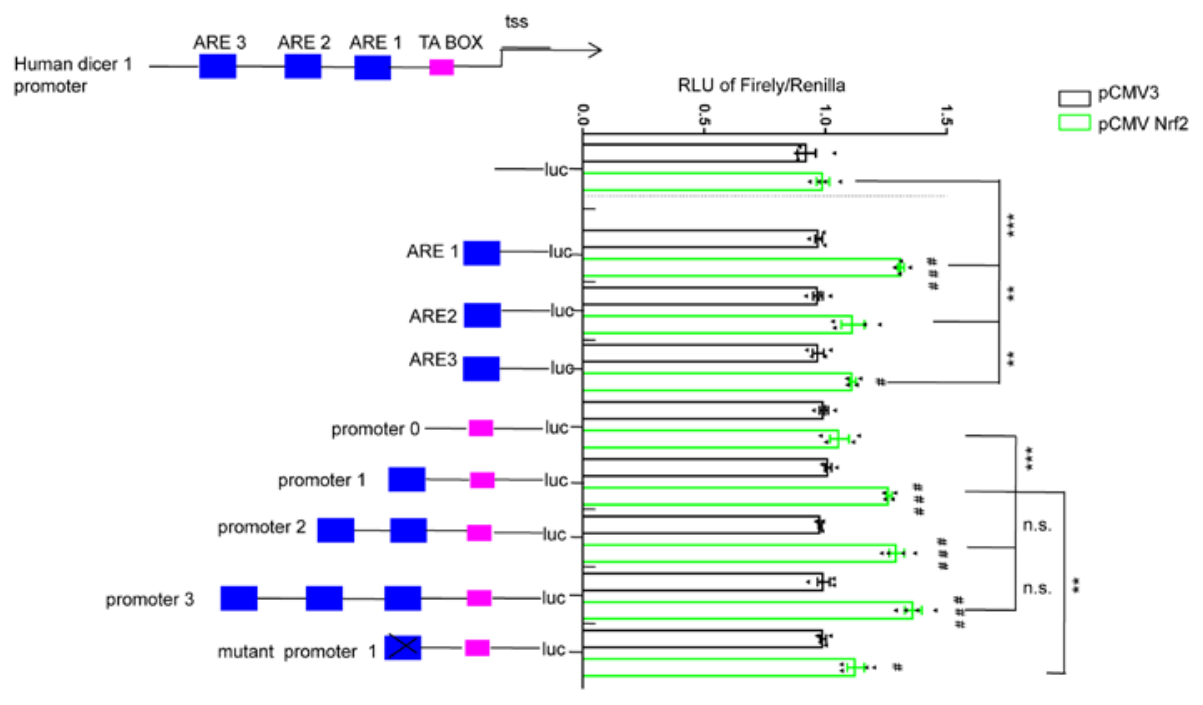

Figure 4. Nrf2 regulated Dicer1 production via Nrf2-ARE pathway. (A) SK-N-BE(2)

cells were left untreated (Blank) or transfected with a human Nrf2-expressing plasmid (pCMV Nrf2, $1.5 \mu \mathrm{g}$ ) or equal amount of vehicle plasmid (pCMV3) in 6-well plates by use of lipofectamine 2000 in DMEM plus 10\% FBS. Forty-eight hours after transfection, the cells were harvested for western blot against Nrf2 and Dicer1. GAPDH was used as a loading control. (B) The protein levels of Dicer1 relative to GAPDH were normalized 
to pCMV group and averaged from three independent culture preparation in A. Oneway ANOVA followed by Tukey's post hoc test was used to compare the differences, pCMV3 vs. pCMV3 Nrf2, ${ }^{*} p=0.0021$. (C) The protein levels of Nrf2/ GAPDH were normalized to pCMV group and averaged from three independent culture preparations in A. One-way ANOVA, followed by Tukey's post hoc test was used to compare the difference, pCMV3 vs. pCMV3 Nrf2, ${ }^{\star \star} p=0.0017$. (D) Neuro-2a cells transfected with a murine Nrf2-expressing (pCMV Nrf2) or vehicle plasmid (pCMV3) by use of lipofectamine 2000 for 48 h. At the end of the culture, the cells were harvested for western blot against Nrf2 and Dicer1. GAPDH was used as a loading control. (E) The protein levels of Dicer1/ GAPDH were normalized to pCMV3 group and averaged from three independent culture preparation in D. Student's t-test was used to compare the differences, pCMV3 vs. pCMV3 Nrf2, ${ }^{\star} p=0.0004$. (F) The protein levels of Nrf2/GAPDH were normalized to pCMV3 group and averaged from three independent culture preparation in D. Student's t-test was used to compare the differences, pCMV3 vs. pCMV3 Nrf2, ${ }^{\star \star *} p=0.024$. (H) The upper panel showed scheme of Dicer 1 gene promoter containing three predicted ARE sites (blue square), TA box (pink red), tss indicating transcriptional start site (tss). HEK293 T cells were seeded at a density of 3 $\mathrm{X} 10^{4}$ cells per well in 48 -well plates. At $\sim 70 \%$ confluency, the cells were transfected with individual construct (150 ng), Renilla luciferase plasmid (7.5 ng), and PCMV3 or pCMV Nrf2 plasmid expressing human Nrf2 (150 ng) by use of lipofectamine 2000 for $48 \mathrm{~h}$. At the end of culture, the cells were harvested and subject to luciferase activity assay. The results were averaged from five independent culture preparation. One-way ANOVA followed by Tukey's post hoc test was used to compare the differences. 
Comparing co-transfection of pCMV3 Nrf2 with co-transfection of pCMV3 in individual construct, ${ }^{\# \#} \mathrm{p}=0.000219$ in ARE1, ${ }^{\#} \mathrm{p}=0.043$ in ARE3, ${ }^{\# \#} \mathrm{p}=0.00047$ in promoter 1 , ${ }^{\# \#} \mathrm{p}=0.000135$ in promoter $2,{ }^{\# \#} \mathrm{p}=0.000114$ in promoter 3 , and ${ }^{\#} \mathrm{p}=0.048$ in mutant promoter 1. Under the condition of co-transfection of pCMV3 Nrf2, ARE1 vs. pGL6-luc, ${ }^{* *} \mathrm{p}=0.00041 ;$ ARE1 vs. ARE2, ${ }^{* \star} \mathrm{p}=0.000825 ;$ ARE1 vs. ARE3, ${ }^{* *} \mathrm{p}=0.0093 ;$ promoter 1 vs. promoter $0,{ }^{* \star *} \mathrm{p}=0.000361$; promoter 1 vs. mutant promoter $1,{ }^{* \star} \mathrm{p}=0.009$, promoter 1 vs. promoter $2, p=0.194$ (n.s); promoter 1 vs. promoter $3, p=0.254$ (n.s). 
A
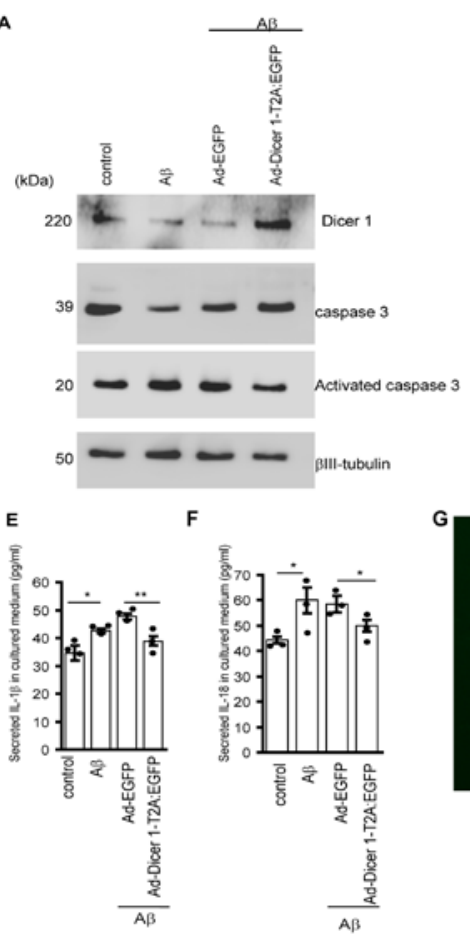

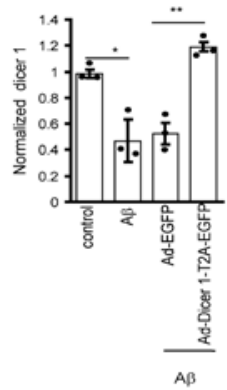

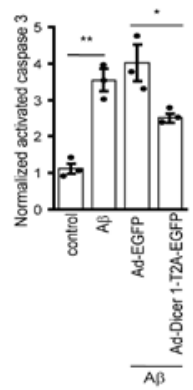

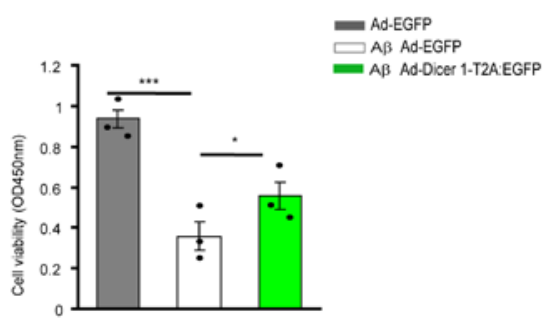

Figure 5. Overexpression of Dicer 1 reduced $A \beta 42$ oligomer-mediated secretion of

\section{IL-1ß and IL-18, apoptosis, and neurite deficit in primary hippocampal neuronal}

cultures. (A) The primary murine hippocampal neurons at DIV 3 were treated with $\mathrm{A} \beta 42$ oligomer $(100 \mathrm{nM})$ for $24 \mathrm{~h}$ or subject to sham treatment in neurobasal medium plus $2 \%$ B27. In a parallel experiment, the medium was replaced with fresh neurobasal medium plus 2\% B27 following $A \beta 42$ treatment, and then infected with vehicle virus, AdEGFP or Ad-dicer1-T2A:EGFP virus $\left(5 \times 10^{7} \mathrm{vg} / \mathrm{mL}\right)$ for $48 \mathrm{~h}$. At the end of treatment, the cultures were harvested and homogenized for western blot against Dicer1, caspase 3, and activated caspase 3. Blll-tubulin was used as a loading control. (B) Dicer1 relative to $\beta I I I-t u b u l i n$ were normalized to sham treatment and averaged from three independent culture preparation. One-way ANOVA followed by Tukey's post hoc test was used to compare the differences. Dicer1 was reduced by $A \beta 42$ oligomer treatment compared to sham treatment $\left({ }^{*} \mathrm{p}=0.033\right)$ and the reduction of Dicer1 by $A \beta 42$ was 
rescued by infection of Ad-dicer1-T2A:EGFP compared to infection by vehicle virus $\left({ }^{\star \star} p=0.0074\right)$. (C) Activated caspase3 relative to $\beta$ III-tubulin were normalized to sham treatment and averaged from three independent culture preparation. One-way ANOVA followed by Tukey's post hoc test was used to compare the differences. Activated caspase 3 was increased by $A \beta 42$ oligomer compared to sham treatment (** $p=0.0061)$, which was reduced by infection of Ad-dicer1-T2A:EGFP compared to infection by vehicle virus ( $\left.{ }^{\star} p=0.023\right)$. (D) The loss of cell viability of hippocampal neurons treated by $A \beta\left({ }^{* \star} p=0.00067\right)$ was significantly rescued by infection with Ad-dicer1-T2A:EGFP compared to infection by vehicle virus $\left({ }^{*} p=0.047\right)$. The values were averaged from quadruplicate cultures with three independent culture preparation. The cultured media were also collected from the cultures treated as in A and subject to ELISA detection of IL-1 $\beta$ and IL-18. (E) $A \beta 42$ oligomer treatment increased IL-1 $\beta$ in the supernatants $\left({ }^{*} p=0.023\right)$ which was reduced by infection of Ad-dicer1-T2A:EGFP compared to infection by vehicle virus $\left({ }^{\star \star} p=0.005\right)$. The values were averaged from quadruplicate cultures with three independent cell preparation and One-way ANOVA followed by Tukey's post hoc test was used to compare the differences. (F) The culture media were collected and subject to ELISA detection of IL-18 as in E. A $\beta$ treatment increased IL-8 $\left({ }^{*} \mathrm{p}=0.019\right)$ which was reduced by infection of Ad-dicer1-T2A:EGFP compared to infection by empty virus $\left({ }^{*} p=0.04\right)$. The values were averaged from quadruplicate cultures with three independent cell preparation and One-way ANOVA followed by Tukey's post hoc test was used to compare the difference. (G)Representative hippocampal neurons (150 neurons for each type of treatment) infected by Ad-EGFP virus (Ad-EGFP), $A \beta 42$ treatment followed by infection with Ad-EGFP virus (Aß Ad- 
EGFP) and $A \beta 42$ treatment followed by infection with Ad-Dicer1-T2A:EGFP virus (A $\beta$

Ad-Dicer1:T2A:EGFP) were indicated. (H) Neurite lengths of hippocampal neurons with treatment from $G$ were calculated and averaged. $A \beta 42$ treatment reduced neurite length compared to sham treatment $\left({ }^{*} \mathrm{p}=0.025\right)$ which was rescued by infection with $\mathrm{Ad}$ dicer 1-T2A:EGFP virus ( $\left.{ }^{*} \mathrm{p}=0.047\right)$. The neurite length averaged from 150 neurons in each treatment and One-way ANOVA followed by Tukey's post hoc test was used to compare the differences. 
A

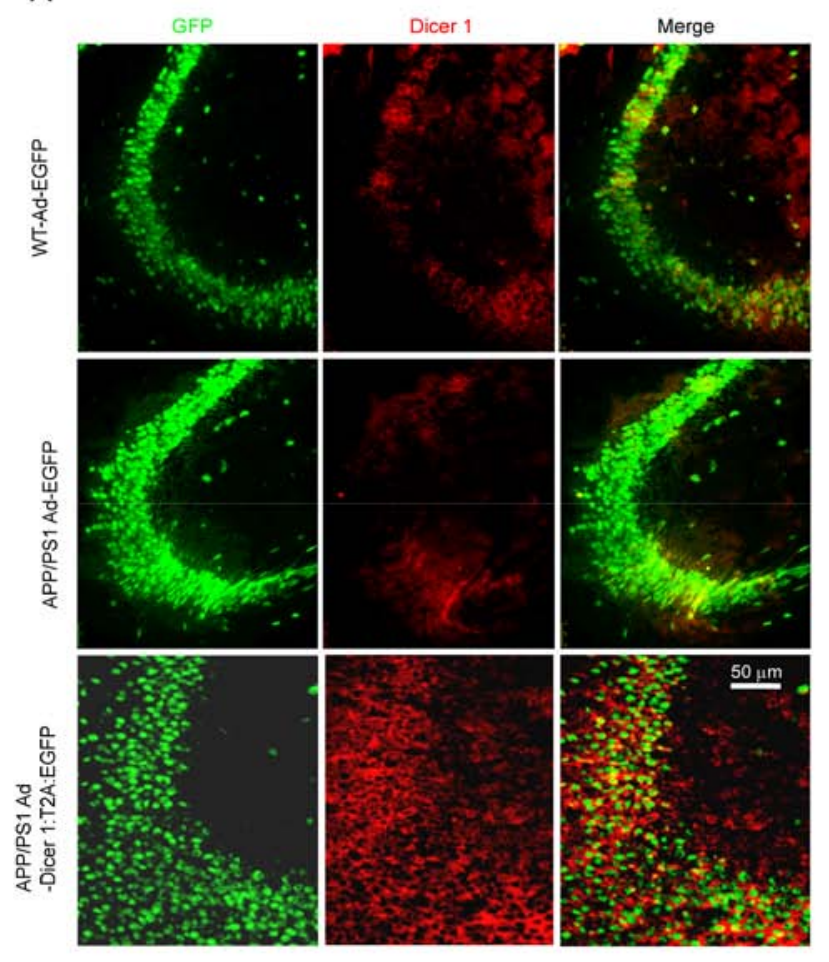

C

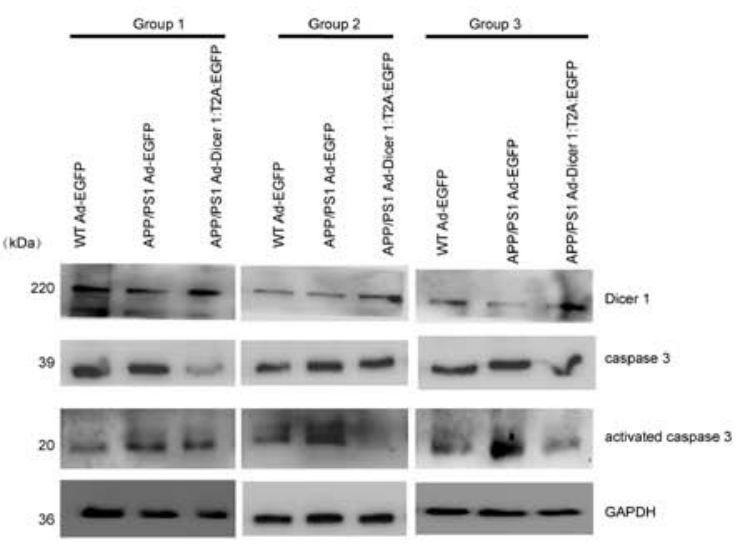

B
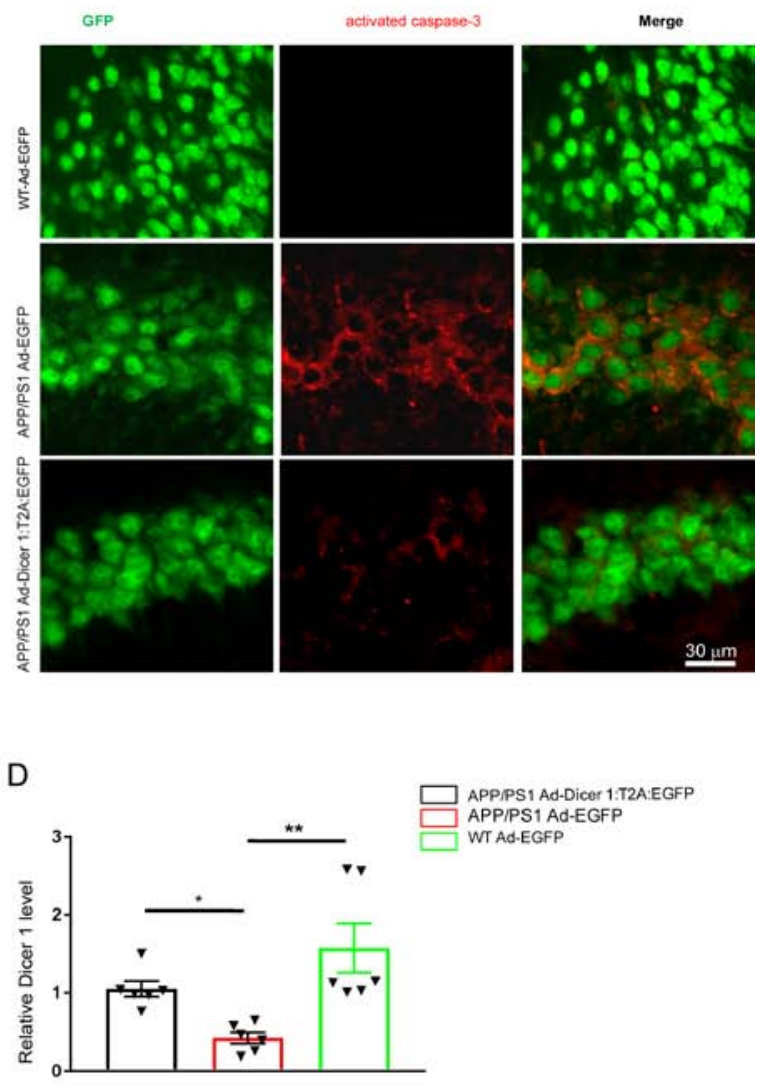

E

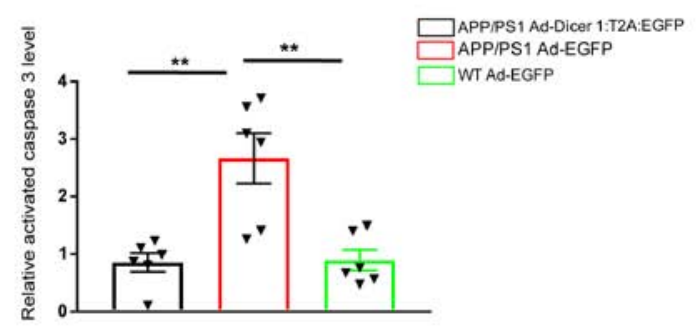

Figure 6. Overexpression of Dicer1 in APPIPS1hippocampus inhibited activated

caspase 3. APP/PS1 mice or WT littermate at the age of 3.5-/4-month were injected with Ad-EGFP or Ad-Dicer1-T2A:EGFP virus $\left(1.2 \times 10^{9} \mathrm{vg} / \mathrm{mL}\right)$ in CA3 hippocampi. 17 days after injection, the injected mice were perfused and each brain was sagittally cut into $\sim 60$ sections at $10 \mu \mathrm{m}$-thick for each section. The sections every other six sections were chosen for double staining of Dicer1 (red) (A) and activated caspase 3 (red) (B). 
The typical images of CA3 hippocampal region were indicated. (A) Dicer1 staining (red) in APP/PS1 CA3 hippocampus was reduced compared to those in WT littermate and infection with Ad-Dicer 1-T2A:EGFP virus dramatically increased Dicer1 staining in CA3 hippocampus. The images were typical of 60 sections from six mice. Bar, $50 \mu \mathrm{m}$. (B) The staining of activated caspase 3 (red) in APP/PS1mice was increased compared to those in WT and this increase in staining was mitigated by infection with Ad-Dicer1T2A:EGFP virus. The images were typical of 60 sections from six mice. Bar, $30 \mu \mathrm{m}$. (C) The CA3 hippocampi were harvested, homogenized, and subjected to centrifugation at $12,000 \times \mathrm{g}$, and the supernatants were used for western blot against Dicer1, caspase3, and activated caspase3, and typical blots were indicated. (D) Dicer1 was reduced in APP/PS1 hippocampi compared to WT ( $\left.{ }^{*} \mathrm{p}=0.0217\right)$, which was increased by infection with Ad-Dicer1-T2A:EGFP virus ( $\left.{ }^{\star \star} \mathrm{p}=0.0093\right)$. The optical densities averaged from six mice in each group and Kruskal-Wallis test was used to compare the differences. (E) Activated caspase 3 relative to GAPDH was increased in APP/PS1 hippocampi compared to WT (** $\mathrm{p}=0.00681)$, which was reduced by infection with AdDicer1-T2A:EGFP virus $\left({ }^{\star \star} \mathrm{p}=0.0067\right)$. The optical densities averaged from six mice in each group and Kruskal-Wallis test was used to compare the differences. 
A

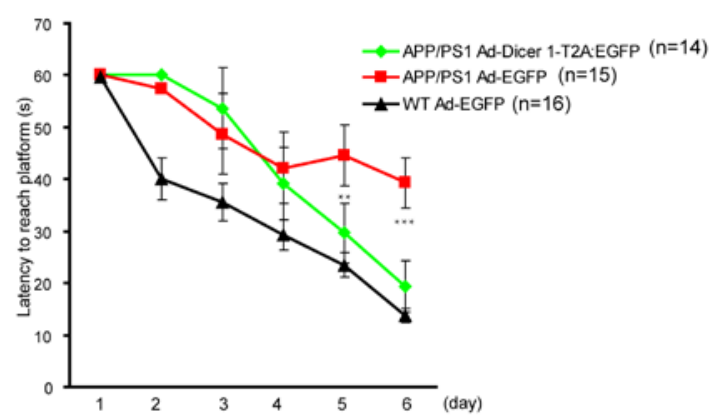

B

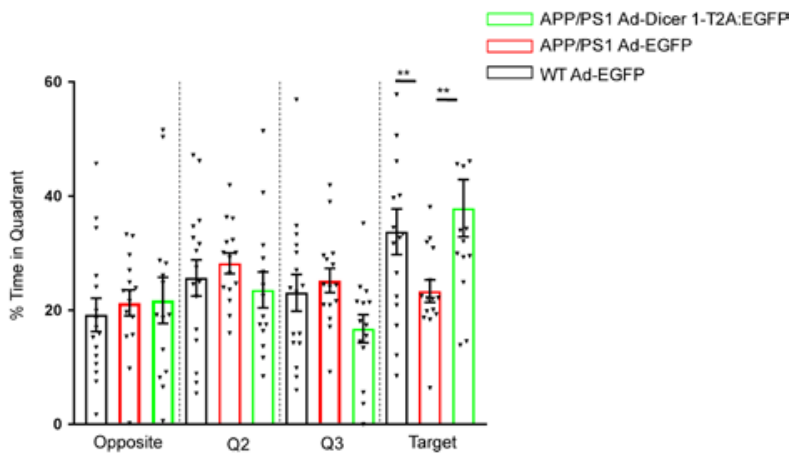

C

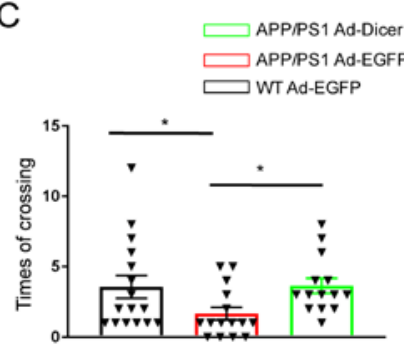

D
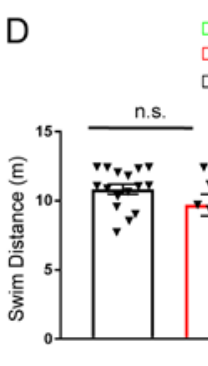

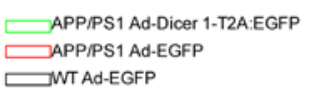

E $\square$ APPIPS1 Ad-Dicer 1-T2A:EGFP TIT Ad-EGFP

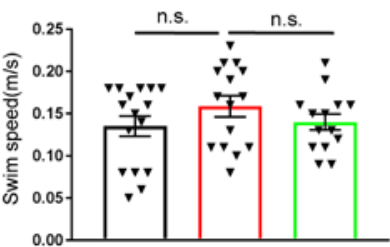

Figure 7. Overexpression of Dicer 1 in CA3 hippocampus significantly improved spatial learning in APP/PS1 mice. Ad-Dicer1-T2A:EGFP virus $\left(1.2 \times 10^{9} \mathrm{vg} / \mathrm{mL}\right)$ or equal amount of Ad-EGFP virus were injected into CA3 hippocampus in APP/PS1or WT mice and behavior test was conducted at 17-23 days after injection. (A) Learning curve of the mice from WT mice injected with Ad-EGFP virus ( $n=16)$, APP/PS1 mice injected with Ad-EGFP virus $(n=15)$ or Ad-Dicer1-T2A:EGFP virus $(n=14)$. The differences of latency to reach platform were analyzed by use of two-way ANOVA with repeated measures against day and treatment (virus injected), $p_{\text {day }}=0.00001, p_{\text {treatment }}=0.0241$, $p_{\text {interaction }}=0.0148$. Tukey's post hoc test was further used to compare the differences between APP/PS1 mice injected with Ad-Dicer1-T2A:EGFP virus and APP/PS1mice injected with Ad-EGFP virus, ${ }^{\star \star} \mathrm{p}=0.008$ on day 5 and ${ }^{* \star \star} \mathrm{p}=0.00001$ on day 6 . (B) Preference of APP/PS1mice for the target quadrant was enhanced by Ad-Dicer 1- 
T2A:EGFP virus injection in CA3 hippocampi. Two-way ANOVA with repeated measures against quadrant and treatment was used to compare the differences, $p_{\text {quadrant }}$ $=0.1122, p_{\text {type }}=1, p_{\text {interaction }}=0.0046$. Tukey's post hoc test was further used to compare the differences among groups, APP/PS1 Ad-Dicer 1-T2A:EGFP ( $n=16)$ vs. APP/PS1 $\operatorname{Ad}-\operatorname{EGFP}(n=15),{ }^{\star \star} \mathrm{p}=0.0087 ;$ APP/PS1 Ad-EGFP $(\mathrm{n}=15)$ vs. WT Ad-EGFP $(\mathrm{n}=16)$, ${ }^{* \star} p=0.0023$. (C) Frequencies of crossing target platform was increased by Dicer1 overexpression. Tukey's post hoc test was further used to compare the differences among groups, APP/PS1 Ad-Dicer 1-T2A:EGFP ( $n=14)$ vs. APP/PS1 Ad-EGFP ( $n=15)$; ${ }^{*} p=0.043 ;$ APP/PS1 Ad-EGFP ( $\left.n=15\right)$ vs. WT Ad-EGFP ( $\left.n=16\right),{ }^{*} p=0.039$. (D) Swim distance and (E) Swim speed did not show differences among groups (n.s). 